\title{
Invariant measures for piecewise convex transformations of an interval
}

\author{
by \\ Christopher Bose (Victoria, BC), \\ Véronique Maume-Deschamps (Dijon), Bernard Schmitt (Dijon) \\ and Sujin Shin (Daejon)
}

\begin{abstract}
We investigate the existence and ergodic properties of absolutely continuous invariant measures for a class of piecewise monotone and convex self-maps of the unit interval. Our assumption entails a type of average convexity which strictly generalizes the case of individual branches being convex, as investigated by Lasota and Yorke (1982). Along with existence, we identify tractable conditions for the invariant measure to be unique and such that the system has exponential decay of correlations on bounded variation functions and Bernoulli natural extension. In the case when there is more than one invariant density we identify a dominant component over which the above properties also hold. Of particular note in our investigation is the lack of smoothness or uniform expansiveness assumptions on the map or its powers.
\end{abstract}

1. Introduction and statement of results. We study nonsingular transformations $T$ from the unit interval $I=[0,1]$ into $I$ that are piecewise monotone and continuous. Specifically, there is a finite partition of $I$ given by $0=a_{0}<a_{1}<\ldots<a_{N}=1$ and such that on each $\left(a_{i-1}, a_{i}\right)$ the restriction $T_{i}=\left.T\right|_{\left(a_{i-1}, a_{i}\right)}$ is continuous and (strictly) increasing. Since each $T_{i}$ is 1-1, they have well defined inverses $\phi_{i}=T_{i}^{-1}$ which may be extended continuously to increasing (and hence a.e. differentiable) functions $\psi_{i}:[0,1] \rightarrow\left[a_{i-1}, a_{i}\right]$, where $\psi_{i}(x)=a_{i-1}$ if $x \leq \inf _{y \in\left(a_{i-1}, a_{i}\right)} T_{i}(y)$; furthermore, $\psi_{i}(x)=a_{i}$ if $x \geq \sup _{y \in\left(a_{i-1}, a_{i}\right)} T_{i}(y)$. Since we are assuming $m \circ T_{i}^{-1} \ll m$ for each $i=1, \ldots, N$, we have

$$
\frac{d m \circ T_{i}^{-1}}{d m}=\psi_{i}^{\prime} \quad m \text {-a.e. }
$$

2000 Mathematics Subject Classification: Primary 37E05, 37A05, 37A25; Secondary $37 \mathrm{~A} 40$.

Research of C. Bose supported by NSERC Grant no. OGP0046586.

Research of S. Shin supported by NSERC Grant no. OGP0046586 and PIMS PDF 1999-2001. 
Throughout this article $m$ denotes the Lebesgue measure on the Borel subsets $\mathcal{B}$ of $[0,1]$.

We consider the Perron-Frobenius operator $P$ on $L^{1}=L^{1}(I, \mathcal{B}, m)$, uniquely defined by the identity

$$
\int P f \cdot g d m=\int f \cdot g \circ T d m \quad \forall f \in L^{1}, g \in L^{\infty} .
$$

In view of our setup we have the following pointwise representation for $P$, taking $g=\chi_{[0, x]}$ : for each $x \in[0,1]$,

$$
\begin{aligned}
\int_{0}^{x} P f(t) d m(t) & =\sum_{i} \int_{T_{i}^{-1}[0, x]} f(s) d m(s) \\
& =\sum_{i} \int_{0}^{x} f \circ \psi_{i}(t) \frac{d m \circ T_{i}^{-1}}{d m}(t) d m(t) \\
& =\int_{0}^{x} \sum_{i} f\left(\psi_{i}(t)\right) \psi_{i}^{\prime}(t) d m(t)
\end{aligned}
$$

from which it follows that $\operatorname{Pf}(x)=\sum_{i=1}^{N} \psi_{i}^{\prime}(x) f\left(\psi_{i}(x)\right)$ for almost every $x \in[0,1]$. Our convexity assumption takes the following form:

(C) Assume that for $i=1, \ldots, N$ there are measurable functions $F_{i}$ : $[0,1] \rightarrow[0, \infty)$ with $F_{i}=\psi_{i}^{\prime} m$-a.e. and such that the family $F_{i}$ satisfies both

(C1) for each $k=1, \ldots, N$ the functions $F_{1}+\ldots+F_{k}$ are decreasing, and

(C2) $F_{1}(0)<1$.

Observe that a branch $T_{i}$ is convex iff its associated $F_{i}$ may be chosen decreasing. Our assumption $(\mathrm{C})$ therefore is strictly weaker than the requirement that each branch be convex.

Under assumption (C) we may again rewrite the pointwise version of (1.1) as

$$
P f(x)=\sum_{i=1}^{N} F_{i}(x) f\left(\psi_{i}(x)\right) \quad \forall f \in L^{1} .
$$

REMARK 1.1. Of course, formula (1.2) requires the following interpretation. Given $f \in L^{1}$, any version of $f$ used on the right hand side of (1.2) will produce a version of $P f$.

TheOREM 1.1. Let $T$ be piecewise monotone and continuous on $I$ as above, and satisfy the convexity condition $(\mathrm{C})$. Then $T$ admits an absolutely continuous invariant probability measure $\nu$, whose density $g=d \nu / d m$ may be chosen to be a decreasing function on $I$. 
REMARK 1.2. There can exist no general result proving existence of a finite a.c.i.m. for an arbitrary piecewise $C^{1}$ expanding interval map. (See [3] where a counterexample is constructed.) Therefore, most existence results in this class depend on additional smoothness conditions on the branches. See, for example, [8], [2] and references cited there.

We note that no uniform continuity assumption on the derivative $T^{\prime}$ is implied by our convexity condition $(\mathrm{C})$, so our investigation is quite different from these classical existence results.

In the shift space setting, P. Hulse [4] has identified a condition on $\mathcal{G}$-functions corresponding to our convexity condition (the terminology is attractive $\mathcal{G}$-function) and has studied the space of attractive $\mathcal{G}$-measures associated to a continuous attractive $\mathcal{G}$-function. In this work, continuity plays a crucial role.

An extensive analysis by Rychlik [11] is closer in spirit to our present investigation, but the uniform expansiveness assumed there is replaced by the weaker form in (C2) which only implies that the branch $T_{1}$ is expanding.

Perhaps closest to our present investigation is an older result of Lasota and Yorke [9] for piecewise convex maps where the main result proved there should be compared to ours. There, all branches are assumed to be convex, and the leftmost branch $T_{1}$ is assumed to satisfy $T^{\prime}(0)>1$. Further, all branches satisfy $T_{1}(0)=T_{2}\left(a_{1}\right)=\ldots=T_{N}\left(a_{N-1}\right)=0$. The assumption of nonsingularity is not necessary as the stronger convexity assumption implies it. Our convexity assumption (C1) is not only weaker than that of LasotaYorke, but is also more natural in the sense that, as we shall see, condition (C1) is necessary and sufficient for the Perron-Frobenius operator $P$ to preserve the class of nonnegative, decreasing functions on $I$ (Lemma 2.2). So, our condition is invariant under taking powers of $T$. Also, this observation leads to a very simple proof of a classical variational inequality, after which the existence of an invariant density can be deduced in the classical manner; see for example [8]. This is discussed in $\S 2$, providing the proof of Theorem 1.1 above.

It is worth noting that if we assume only monotonicity of the branches of $T$ along with the convexity condition (C) then it appears that most of our analysis fails to go through. In particular the basic result in Lemma 2.2 concerning invariance of the cone of positive decreasing functions (upon which everything which follows depends) fails to hold.

In [9], Lasota-Yorke convex maps are shown to have the property that there is a unique invariant probability density $g$ and that the a.c.i.p.m. $d \mu=g d x$ is exact for $T$. This is not the case for maps satisfying our weaker convexity condition. In $\S 4$ and $\S 5$ we identify a dominant component for a given decreasing invariant density and prove uniqueness and exactness of this 
dominant component (an interval). Also, in $\S 4$ we prove exponential decay of correlations, the uniform expanding property and the Bernoulli property when the dominant component is not normalized Lebesgue measure. This restriction is equivalent to the requirement that $T$ be expanding at the rightmost endpoint of the dominant component, which we call condition (E), for expanding. We note that exponential decay of correlations and the uniform expanding property were proved for Lasota-Yorke convex maps in [5].

The remaining question of when there exists a unique a.c.i.m. for $T$ is discussed in $\S 6$. We identify a mixing condition $(\mathrm{M})$ which ensures that there is exactly one invariant density in BV and the resulting system $(T, g d x)$ is exact. Again, if the expanding condition (E) is also satisfied, then some power of $T$ is expanding and $T$ is Bernoulli with respect to its unique a.c.i.m. Furthermore, Lasota-Yorke convex maps satisfy our condition (M), but (M) does not imply that some power of $T$ is uniformly expanding, so this final section identifies a proper extension of the results in [9].

Much of our argument depends on the identification of suitable invariant cones for the operator $P$ and the construction of norms equivalent to the bounded-variation norm from these cones. We give a brief discussion of these matters in $\S 3$. The reader looking for more complete background on this method should consult R. Murray [10] where many of the omitted details may be found.

The first author is pleased to acknowledge the hospitality of the Laboratoire de Topologie, Université de Bourgogne during the Fall of 1996 where first the idea to revisit the Lasota-Yorke maps from a more current point of view was proposed.

2. Existence of an invariant density. Throughout this section $T$ is assumed to satisfy the conditions of Theorem 1.1.

Lemma 2.1. Without loss of generality, we may assume that for $k=$ $1, \ldots, N$ the functions $F_{1}+\ldots+F_{k}$ are decreasing, and upper semicontinuous on $[0,1]$.

Proof. Every decreasing function on $[0,1]$ can be modified on a set of measure zero to be upper semicontinuous and decreasing. Apply this inductively for $k=1, \ldots, N$. Changing each of the functions $F_{i}$ on sets of measure zero will not change the operator $P$.

REMARK 2.1. The simple result above leads to a kind of uniqueness in the pointwise representation of the Perron-Frobenius operator. Suppose $F f(x)=\sum_{i=1}^{N} F_{i}(x) f\left(\psi_{i}(x)\right)$ while $G f(x)=\sum_{i=1}^{N} G_{i}(x) f\left(\psi_{i}(x)\right)$ for all $f \in L^{1}$ are two Perron-Frobenius operators with weight functions $\left\{F_{i}\right\}$, $\left\{G_{i}\right\}$ both satisfying $(\mathrm{C} 1)$. Suppose $F f=G f$ for all $f \in L^{1}$. If both sets of 
weight functions have upper semicontinuous sums as in the above lemma, then $F_{i}=G_{i}$ on $(0,1]$ since two upper semicontinuous, decreasing functions which agree almost everywhere must be identical except possibly at zero. Now it is a simple matter to change the definition of the $F_{i}$ at the single point zero, still maintaining condition $(\mathrm{C} 1)$ so that $F_{i}=G_{i}$ on $[0,1]$. We will have a number of opportunities in the following arguments to make use of this simple observation.

REMARK 2.2. In a similar vein, suppose $c \in\left(a_{i-1}, a_{i}\right)$ for some $i=$ $1, \ldots, N$. Define

$$
T_{j}(x)= \begin{cases}T_{j}(x) & \text { if } 1 \leq j<i \\ T_{i}(x) & \text { if } j=i \text { and } x \in\left[a_{i-1}, c\right], \\ T_{i}(x) & \text { if } j=i+1 \text { and } x \in\left[c, a_{i}\right] \\ T_{j-1}(x) & \text { if } i+1<j \leq N+1\end{cases}
$$

in other words, we split the $i$ th branch into two subbranches at the point $c$. Then this new $T$ with $N+1$ branches still satisfies the convexity condition $(\mathrm{C})$ and generates the same operator $P$, although the pointwise representation (1.2) will be changed.

Let $\mathcal{J}=\{f:[0,1] \rightarrow[0, \infty) \mid f(x) \geq f(y)$ whenever $x \leq y\}$ be the cone of nonnegative, decreasing functions on $I$. As a further consequence of the convexity condition $(\mathrm{C} 1)$ we have

Lemma 2.2. A necessary and sufficient condition for an operator of the form

$$
\operatorname{Pf}(x)=\sum_{i=1}^{N} F_{i}(x) f\left(\psi_{i}(x)\right), \quad F_{i} \geq 0,
$$

to satisfy $P: \mathcal{J} \rightarrow \mathcal{J}$ is that the $F_{i}$ 's satisfy condition $(\mathrm{C} 1)$.

Observe that $T(0)=0$ since if $T(0)=a>0$ then $F_{1}(0)=0$ and condition (C1) implies $F_{1} \equiv 0$. But then $a_{1}=\int_{0}^{1} F(t) d t=0$, which is a contradiction.

COROLlary 2.3. The convexity condition $(\mathrm{C})$ is preserved under powers of $T$.

Proof of Lemma 2.2. Let $f \in \mathcal{J}$ and $x \leq y$. Define $x_{i}=\psi_{i}(x)$ and $y_{i}=\psi_{i}(y) \geq x_{i}$ for $i=1, \ldots, N$. Then

$$
\begin{aligned}
\operatorname{Pf}(x)-P f(y) & =\sum_{i=1}^{N}\left[F_{i}(x) f\left(x_{i}\right)-F_{i}(y) f\left(y_{i}\right)\right] \\
& \geq \sum_{i=1}^{N}\left(F_{i}(x)-F_{i}(y)\right) f\left(x_{i}\right)
\end{aligned}
$$

since $F_{i} \geq 0$ and $f\left(x_{i}\right) \geq f\left(y_{i}\right)$. 
Define the following $N$-dimensional vectors:

$$
\begin{aligned}
\overrightarrow{\Delta F} & =\left\langle F_{i}(x)-F_{i}(y)\right\rangle \in \mathbb{R}^{N}, \\
\vec{f} & =\left\langle f\left(x_{i}\right)\right\rangle \in \text { interior of positive cone of } \mathbb{R}^{N}, \\
\vec{b}_{j} & =\langle\underbrace{1, \ldots, 1}_{j \text { times }}, 0, \ldots, 0\rangle, \quad j=1, \ldots, N .
\end{aligned}
$$

With this notation we rewrite (2.1) simply as

$$
\operatorname{Pf}(x)-\operatorname{Pf}(y) \geq \overrightarrow{\Delta F} \cdot \vec{f} .
$$

The convexity assumption (C1) implies

$$
\overrightarrow{\Delta F} \cdot \vec{b}_{j} \geq 0, \quad j=1, \ldots, N .
$$

Furthermore,

$$
\vec{f}=\sum_{j=1}^{N-1}\left(f\left(x_{j}\right)-f\left(x_{j+1}\right)\right) \vec{b}_{j}+f\left(x_{N}\right) \vec{b}_{N},
$$

with all coefficients in this expression nonnegative since $f \in \mathcal{J}$. By (2.3), (2.4), linearity in (2.2) implies $P f \in \mathcal{J}$.

As for the converse, note that the inequality at (2.1) is sharp on $\mathcal{J}$, that is, if the $F_{i}$ fail to satisfy the convexity assumption (C1), then there exists an $f$ in $\mathcal{J}$ with $\operatorname{Pf} \notin \mathcal{J}$.

If $f:[0,1] \rightarrow \mathbb{R}$ we denote the variation of $f$ by $\mathrm{V}_{I} f$ and let BV denote the Banach space of bounded variation functions (with norm $\|f\|_{\mathrm{BV}}=\mathrm{V}_{I} f+$ $\left.\|f\|_{1}\right)$. As in the classical situation, we seek a variational inequality for $P$ in order to establish compactness of the sequence of iterates of a function in BV. We have been unable to prove such an inequality on all of BV, but following below is the inequality on the subcone $\mathcal{J} \subset \mathrm{BV}$, and this will turn out to be sufficient for our purposes.

Lemma 2.4. For each a satisfying $F_{1}(0)<a<1$ there exists a constant $b=b(a)<\infty$ so that for all $f \in \mathcal{J}$,

$$
\mathrm{V}_{I} P f \leq a \mathrm{~V}_{I} f+b\|f\|_{1} .
$$

Proof. We first note that there exists a weak variational inequality: there exist positive constants $A, B<\infty$ such that

$$
\mathrm{V}_{I} \text { Pf } \leq A \mathrm{~V}_{I} f+B\|f\|_{1} \quad \forall f \in \mathcal{J} .
$$

For $f \in \mathcal{J}$,

$$
\begin{aligned}
\operatorname{Pf}(0)-\operatorname{Pf}(1) & =\sum_{i=1}^{N} F_{i}(0) f\left(a_{i-1}\right)-\sum_{i=1}^{N} F_{i}(1) f\left(a_{i}\right) \\
& \leq\left(\sum_{i=1}^{N} F_{i}(0)\right) f(0)-\left(\sum_{i=1}^{N} F_{i}(1)\right) f(1)=: \Gamma f(0)-\gamma f(1)
\end{aligned}
$$


Note, by condition (C1), $\Gamma \geq \gamma$, and for $f \in \mathcal{J}, f(1) \leq\|f\|_{1}$, so we obtain

$$
\mathrm{V}_{I} P f \leq \Gamma \mathrm{V}_{I} f+(\Gamma-\gamma)\|f\|_{1} .
$$

This shows we may set $A=\Gamma$ and $B=\Gamma-\gamma$ in our first variational inequality. It also shows that the lemma is true on the subspace of constant functions $\{c 1\}_{c \geq 0}$, so we may restrict our attention to $\mathcal{J}-\{c 1\}_{c \geq 0}$.

Suppose the lemma is false. Then there is a number $\widehat{a}$, with $F_{1}(0)<$ $\widehat{a}<1$, and sequences $0 \neq f_{n} \in \mathcal{J}, \mathrm{V}_{I} f_{n} \neq 0$, and $A_{n}>0, \lim _{n} A_{n}=\infty$, satisfying

$$
\frac{\mathrm{V}_{I} P f_{n}-\widehat{a} \mathrm{~V}_{I} f_{n}}{\left\|f_{n}\right\|_{1}}=A_{n} .
$$

Since the left hand side of (2.5) is invariant under $f \mapsto c f, c>0$, we may assume $\mathrm{V}_{I} f_{n}=1$ for all $n$. Using the weak variational inequality established above, we have

$$
\frac{A+B\left\|f_{n}\right\|_{1}-\widehat{a}}{\left\|f_{n}\right\|_{1}} \geq A_{n} \rightarrow \infty .
$$

The left hand side above is uniformly bounded on sets where $\left\|f_{n}\right\|_{1} \geq \delta>0$, so we must have $\left\|f_{n}\right\|_{1} \rightarrow 0$. Dropping to a subsequence if necessary, we may assume $f_{n} \rightarrow 0$ for $m$-a.e. $x \in I$. Choose $x_{0} \in\left(0, a_{1}\right)$ satisfying $f_{n}\left(x_{0}\right) \rightarrow 0$. Let $\delta>0$ be fixed, and pick $n_{0}$ so that $f_{n_{0}}\left(x_{0}\right)<\delta$. Then we have $f_{n_{0}}\left(a_{i}\right)$ $<\delta, i=1, \ldots, N, f_{n_{0}}(0)<1+\delta$, and we may make the estimate

$$
\begin{aligned}
P f_{n_{0}}(0)-P f_{n_{0}}(1) & \leq P f_{n_{0}}(0) \leq \sum_{i=1}^{N} F_{i}(0) f_{n_{0}}\left(a_{i-1}\right) \\
& \leq F_{1}(0)(1+\delta)+\left(\sum_{i=2}^{N} F_{i}(0)\right) \delta \\
& =F_{1}(0)+\left(\sum_{i=1}^{N} F_{i}(1)\right) \delta<\widehat{a}
\end{aligned}
$$

provided $\delta$ was chosen sufficiently small. Thus $\mathrm{V}_{I} P f_{n_{0}}<\widehat{a} \mathrm{~V}_{I} f_{n_{0}}$, which contradicts the sign in (2.5).

Proof of Theorem 1.1. Applying the previous lemma iteratively to the constant function $\mathbf{1}$ we obtain

$$
\mathrm{V}_{I} P^{s} \mathbf{1} \leq b\left(1+a+a^{2}+\ldots+a^{s-1}\right) \leq b(1-a)^{-1} .
$$

Now consider the sequence $g_{n}=(1 / n) \sum_{s=0}^{n-1} P^{s} \mathbf{1}$. The following properties are now evident:

(1) $g_{n} \in \mathcal{J}$ and $\left\|g_{n}\right\|_{1}=\int g_{n}=1, n \geq 1$.

(2) $\left\|P g_{n}-g_{n}\right\|_{1} \rightarrow 0$ as $n \rightarrow \infty$ since $\sup \left\|P^{s} \mathbf{1}\right\|_{\infty}<\infty$.

(3) The sequence $\left\{g_{n}\right\}$ is relatively compact in $L^{1}$ by Helly's Theorem. 
(4) If, in $L^{1}$ norm, $g=\lim _{k} g_{n_{k}}$ for some subsequence, then $g \geq 0$, $P g=g$, and $\|g\|_{1}=1$.

Finally, since $g$ may be obtained as the $L^{1}$ limit of a sequence of decreasing functions, we may, by an elementary argument, find a version of $g$ which is decreasing.

3. Preliminaries about cones and norms equivalent to $\|\cdot\|_{B V}$. Recall that $\mathcal{J}=\{f:[0,1] \rightarrow[0, \infty) \mid f$ is decreasing $\}$. Given a function $f$ on $I=[0,1]$, we will simply denote $\mathrm{V}_{I} f$ by $\mathrm{V} f$. We denote by $\mathrm{BV}_{0}$ the (Banach) subspace of bounded variation functions which integrate to zero. We reserve $\|\cdot\|$ for the $L^{1}$ norm $\|\cdot\|_{1}$. Of course for a given $f \in \mathrm{BV}$, there exist $f^{1}, f^{2} \in \mathcal{J}$ such that $f=f^{1}-f^{2}$. In fact the following is also true.

LEMMA 3.1. Given $f \in \mathrm{BV}$, there exist $f^{1}, f^{2} \in \mathcal{J}$ such that

(1) $f=f^{1}-f^{2}$;

(2) $\mathrm{V} f=\mathrm{V} f^{1}+\mathrm{V} f^{2}$;

(3) if $f=g-h \in \mathrm{BV}$ and $g, h \in \mathcal{J}$, then $\mathrm{V} f^{1} \leq \mathrm{Vg}$ and $\mathrm{V} f^{2} \leq \mathrm{V} h$ and $\left\|f^{1}\right\|+\left\|f^{2}\right\| \leq\|g\|+\|h\|$. Furthermore, if $g \neq f^{1}\left(\right.$ so $\left.h \neq f^{2}\right)$, then $\left\|f^{1}\right\|+\left\|f^{2}\right\|<\|g\|+\|h\|$.

Proof. For each $x \in I$, let $T_{f}(x)=\mathrm{V}_{0}^{x} f$. Define $f^{1}$ and $f^{2}$ by

$$
f^{1}=\frac{1}{2}\left(\mathrm{~V} f+|f(1)|+f-T_{f}\right) \quad \text { and } \quad f^{2}=\frac{1}{2}\left(\mathrm{~V} f+|f(1)|-f-T_{f}\right),
$$

so clearly $f=f^{1}-f^{2}$. It is easy to check that $f^{1}$ and $f^{2}$ are decreasing. Since $f^{1}(1)=\frac{1}{2}\{|f(1)|+f(1)\} \geq 0$, we have $f^{1} \geq 0$ and similarly $f^{2} \geq 0$. Thus $f^{1}, f^{2} \in \mathcal{J}$. Also

$$
\begin{aligned}
\mathrm{V} f^{1}+\mathrm{V} f^{2} & =f^{1}(0)-f^{1}(1)+f^{2}(0)-f^{2}(1) \\
& =\frac{1}{2}(f(0)-f(1)+\mathrm{V} f)+\frac{1}{2}(f(1)-f(0)+\mathrm{V} f)=\mathrm{V} f .
\end{aligned}
$$

Suppose $f=g-h$ with $g, h \in \mathcal{J}$. Then

$$
\begin{aligned}
\mathrm{V} g+\mathrm{V} h & =\mathrm{V} g+[h(0)-h(1)]=\mathrm{V} g+[g(0)-f(0)-g(1)+f(1)] \\
& =2 \mathrm{~V} g-f(0)+f(1) .
\end{aligned}
$$

In particular, $\mathrm{V} f^{1}+\mathrm{V} f^{2}=2 \mathrm{~V} f^{1}-f(0)+f(1)=2 \mathrm{~V} f^{1}-\mathrm{V} g+\mathrm{V} h$. This implies that $\mathrm{V} f^{1} \leq \mathrm{V} g$, since $\mathrm{V} f^{1}+\mathrm{V} f^{2}=\mathrm{V} f \leq \mathrm{V} g+\mathrm{V} h$. Similarly, we have $\mathrm{V} f^{2} \leq \mathrm{Vh}$.

To see $\left\|f^{1}\right\|+\left\|f^{2}\right\| \leq\|g\|+\|h\|$, notice that for each $x \in I$,

$$
\begin{aligned}
0 & \leq \mathrm{V} f-T_{f}(x)=\mathrm{V}_{0}^{1} f-\mathrm{V}_{0}^{x} f \leq \mathrm{V}_{x}^{1} f \leq \mathrm{V}_{x}^{1} g+\mathrm{V}_{x}^{1} h \\
& =g(x)-g(1)+h(x)-h(1)
\end{aligned}
$$

and hence

$$
\mathrm{V} f-\left\|T_{f}\right\|=\left\|\mathrm{V} f-T_{f}\right\| \leq\|g\|+\|h\|-g(1)-h(1) .
$$


Since $|f(1)| \leq g(1)+h(1)$, it follows that

$$
\begin{aligned}
\left\|f^{1}\right\| & +\left\|f^{2}\right\| \\
& =\frac{1}{2}\left[\int\left(\mathrm{V} f+|f(1)|+f-T_{f}\right)+\int\left(\mathrm{V} f+|f(1)|-f-T_{f}\right)\right] \\
& =\mathrm{V} f+|f(1)|-\left\|T_{f}\right\| \leq\|g\|+\|h\| .
\end{aligned}
$$

Finally suppose $\left\|f^{1}\right\|+\left\|f^{2}\right\|=\|g\|+\|h\|$. It follows from (3.1) and (3.2) that $|f(1)|=g(1)+h(1)$, which implies that for each $x \in I$,

$$
\begin{aligned}
f^{1}(x)+f^{2}(x) & =\mathrm{V} f+|f(1)|-T_{f}(x)=g(x)+h(x)-g(1)-h(1)+|f(1)| \\
& =g(x)+h(x),
\end{aligned}
$$

i.e., $f^{1}+f^{2}=g+h$. Since $f^{1}-f^{2}=g-h$, we have $g=f^{1}$ and $h=f^{2}$.

Definition 3.1. For a given $f \in \mathrm{BV}$, with $f^{1}, f^{2}$ defined as above, we will call $f=f^{1}-f^{2}$ the variational decomposition of $f$.

Notice that $\mathcal{J}$ is an $\mathbb{R}^{+}$-module, i.e., if $f, g \in \mathcal{J}$, then $f+g \in \mathcal{J}$ and cf $\in \mathcal{J}$ for any $c \in \mathbb{R}^{+}$. We now introduce a class of submodules (or cones) of $\mathcal{J}$.

Definition 3.2. For a given $K>0$, define $\mathcal{C}_{K}$ by

$$
\mathcal{C}_{K}=\{f \in \mathrm{BV} \mid f \in \mathcal{J} \text { and } \mathrm{V} f \leq K\|f\|\} .
$$

From each $\mathcal{C}_{K}, K>0$, we may construct a vector space $\Gamma_{K}$ of functions on $I$ via

$$
\begin{aligned}
& \Gamma_{K}=\left\{f \in \mathrm{BV} \mid \text { there exist } f^{1}, f^{2} \in \mathcal{C}_{K}\right. \\
& \text { such that } \left.f=f^{1}-f^{2} \text { and }\left\|f^{1}\right\|=\left\|f^{2}\right\|\right\} .
\end{aligned}
$$

On $\Gamma_{K}$ define $\|\cdot\|_{\Gamma_{K}}$ as follows:

$$
\|f\|_{\Gamma_{K}}=\inf \left\{\left\|f^{1}\right\| \mid f=f^{1}-f^{2}, \text { where } f^{1}, f^{2} \in \mathcal{C}_{K} \text { and }\left\|f^{1}\right\|=\left\|f^{2}\right\|\right\} .
$$

We collect some basic facts about these objects.

Lemma 3.2. For each $K>0$, the following hold:

(1) $\Gamma_{K}$ with $\|\cdot\|_{\Gamma_{K}}$ is a normed vector space. (In fact it is a Banach space.)

(2) $f \in \Gamma_{K}$ if and only if $f \in \mathrm{BV}_{0}$.

(3) If $f=f^{1}-f^{2}$ is the variational decomposition of $f \in \mathrm{BV}_{0}$, then there exists $c \geq 0$ such that $f^{1}+c, f^{2}+c \in \mathcal{C}_{K}$ and $\|f\|_{\Gamma_{K}}=\left\|f^{1}+c\right\|$.

(4) For a given $f \in \mathrm{BV}_{0}$,

$$
\min \{1, K\}\|f\|_{\Gamma_{K}} \leq\|f\|_{\mathrm{BV}} \leq 2(K+1)\|f\|_{\Gamma_{K}} .
$$

In particular, all the norms $\|\cdot\|_{\Gamma_{K}}$ are equivalent, and equivalent to $\|\cdot\|_{\mathrm{BV}}$ on $\mathrm{BV}_{0}$. 
Proof. (1) It is an elementary check that $\Gamma_{K}$ is a vector space and that $\|\cdot\|_{\Gamma_{K}}$ is a norm on it. Completeness follows from (2) and (4) below.

(2) If $f \in \Gamma_{K}$, then by definition there exist $f^{1}, f^{2} \in \mathcal{C}_{K}$ such that $f=f^{1}-f^{2}$ and $\left\|f^{1}\right\|=\left\|f^{2}\right\|$. Since $\int f=\left\|f^{1}\right\|-\left\|f^{2}\right\|=0$, it follows that $f \in \mathrm{BV}_{0}$.

Conversely, suppose $f \in \mathrm{BV}_{0}$ and $f=f^{1}-f^{2}$ is the variational decomposition of $f$. Since $0=\int f=\left\|f^{1}\right\|-\left\|f^{2}\right\|$, we have $\left\|f^{1}\right\|=\left\|f^{2}\right\|$. Let

$$
\alpha=\frac{1}{K} \max \left\{\mathrm{V} f^{1}, \mathrm{~V} f^{2}\right\}-\left\|f^{1}\right\| .
$$

If $\alpha<0$, then $\mathrm{V} f^{1} \leq K\left\|f^{1}\right\|$ and $\mathrm{V} f^{2} \leq K\left\|f^{1}\right\|=K\left\|f^{2}\right\|$, so $f^{1}, f^{2} \in \mathcal{C}_{K}$. Thus $f \in \Gamma_{K}$. If $\alpha \geq 0$, then it is easy to check that $f^{1}+\alpha, f^{2}+\alpha \in \mathcal{C}_{K}$. Since $f=\left(f^{1}+\alpha\right)-\left(f^{2}+\alpha\right)$ and $\left\|f^{1}+\alpha\right\|=\left\|f^{2}+\alpha\right\|$, it follows that $f \in \Gamma_{K}$.

(3) Let $f \in \mathrm{BV}_{0}$ and $f=f^{1}-f^{2}$ be the variational decomposition of $f$. Let $\alpha$ be given as above and $c=\max \{\alpha, 0\}$. It follows from the proof of (2) that $f^{1}+c, f^{2}+c \in \mathcal{C}_{K}$. If $\alpha<0$, i.e., $c=0$, then it is clear that $\|f\|_{\Gamma_{K}}=\left\|f^{1}\right\|=\left\|f^{1}+c\right\|$ (see Lemma 3.1). Now suppose $c=\alpha \geq 0$. If $f=g-h$ and $g, h \in \mathcal{C}_{K} \subset \mathcal{J}$, then it follows from Lemma 3.1 that $\mathrm{V} f^{1} \leq \mathrm{V} g$ and $\mathrm{V} f^{2} \leq \mathrm{V} h$. Thus

$$
\begin{aligned}
\left\|f^{1}+c\right\| & =\left\|f^{1}\right\|+c=\frac{1}{K} \max \left\{\mathrm{V} f^{1}, \mathrm{~V} f^{2}\right\} \leq \frac{1}{K} \max \{\mathrm{V} g, \mathrm{~V} h\} \\
& \leq \frac{1}{K} \max \{K\|g\|, K\|h\|\}=\|g\| .
\end{aligned}
$$

Since $f^{1}+c, f^{2}+c \in \mathcal{C}_{K}$, it follows that $\|f\|_{\Gamma_{K}}=\left\|f^{1}+c\right\|$. Note that there is at most one value of $c$ which can satisfy the previous identity.

(4) Let $f=f^{1}-f^{2}$ be the variational decomposition of $f$ and let $c$ be as in (3) so that $\|f\|_{\Gamma_{K}}=\left\|f^{1}+c\right\|$. Assume that $c=\alpha \geq 0$ and make the estimate

$$
\left\|f^{1}+c\right\|=\left\|f^{1}\right\|+c=\frac{1}{K} \max \left\{\mathrm{V} f^{1}, \mathrm{~V} f^{2}\right\} \leq \frac{1}{K} \mathrm{~V} f \leq \frac{1}{K}\|f\|_{\mathrm{BV}} .
$$

On the other hand, if $c=0$ and $\alpha<0$ we proceed as follows. Notice that either $f^{1}(1)=0$ or $f^{2}(1)=0$ for otherwise we could subtract a small multiple of the identity from both, contradicting Lemma 3.1(3). Assuming the first case (the other is identical) we estimate

$$
\left\|f^{1}+c\right\|=\left\|f^{1}\right\| \leq\|f\|_{\infty} \leq \mathrm{V} f^{1} \leq \mathrm{V} f \leq\|f\|_{\mathrm{BV}} .
$$

This proves the first inequality in (4). For the other inequality, by the proof of (3) with $c$ defined as above

$$
\begin{aligned}
\|f\|_{\mathrm{BV}} & =\mathrm{V} f+\|f\|=\mathrm{V}\left(f^{1}+c\right)+\mathrm{V}\left(f^{2}+c\right)+\|f\| \\
& \leq K\left\|f^{1}+c\right\|+K\left\|f^{2}+c\right\|+\left\|f^{1}\right\|+\left\|f^{2}\right\| \\
& \leq 2(K+1)\left\|f^{1}+c\right\|=2(K+1)\|f\|_{\Gamma_{K}} .
\end{aligned}
$$


REMARK 3.1. We remark that the above construction follows closely the setup in [10], although our choice of the basic cones $\mathcal{C}_{K}$ is different, leading to some changes in the proofs and to some of the constants in the estimates.

Using Lemma 2.4, choose $a$ and $b<\infty$ so that $F_{1}(0)<a<1$ and $\mathrm{VP} f \leq a \mathrm{~V} f+b\|f\|$ for any $f \in \mathcal{J}$. It inductively follows that for any given $f \in \mathcal{J}$ and for each $m \geq 1$,

$$
\mathrm{V} P^{m} f \leq a^{m} \mathrm{~V} f+\frac{b\left(1-a^{m}\right)}{1-a}\|f\| .
$$

Lemma 3.3. For a given $K \geq b /(1-a), P$ preserves $\mathcal{C}_{K}$, i.e., $P$ maps $\mathcal{C}_{K}$ into $\mathcal{C}_{K}$.

Proof. Let $K \geq b /(1-a)$. If $f \in \mathcal{C}_{K}$, then

$$
\mathrm{VPf} \leq a \mathrm{~V} f+b\|f\| \leq a K\|f\|+(1-a) K\|f\|=K\|f\|=K\|P f\|,
$$

which shows $\operatorname{Pf} \in \mathcal{C}_{K}$.

REMARK 3.2. Let $S_{1}$ denote the unit sphere in $L^{1}$. Each subset $\mathcal{C}_{K} \cap S_{1}$ is a convex and compact subset of $L^{1}$. By Lemma 3.3 and the Markov property for $P$, for all sufficiently large $K$ each of these subsets is preserved by $P$. By the Schauder-Tikhonov Theorem, $P$ will have a fixed point in $\mathcal{C}_{K} \cap S_{1}$. This gives another proof of the existence of a decreasing invariant probability density, as was already derived at the end of $\S 2$.

4. Ergodic properties of an invariant measure: case I. The techniques developed in the previous section will now be used to study the question of ergodic properties. Throughout this section $T$ is assumed to satisfy the conditions of Theorem 1.1. For each $k=1, \ldots, N$, let $\mathcal{F}_{k}$ denote $\mathcal{F}_{k}=\sum_{i=1}^{k} F_{i}$. From Lemma 2.1, without loss of generality, we may assume that for each $k=1, \ldots, N, \mathcal{F}_{k}$ is upper semicontinuous. Also, for a given closed interval $[c, d] \subseteq[0,1](c<d)$, we define $T[c, d]$ to be

$$
T[c, d]=\overline{T(c, d)}=\bigcup_{j=1}^{N} T_{j}\left(\overline{\left[a_{j-1}, a_{j}\right] \cap(c, d)}\right) .
$$

Consequently, $T[c, d]$ is a finite union of nontrivial closed intervals. Under this notation, the following holds.

Lemma 4.1. For each $k=0,1, \ldots, T^{k}\left[0, a_{1}\right]$ is a closed interval containing $\left[0, a_{1}\right]$. Moreover, $T^{k}\left[0, a_{1}\right] \subseteq T^{k+1}\left[0, a_{1}\right]$ so that we have an increasing sequence of closed intervals.

Proof. The first statement is obviously true for $k=0$. Suppose $T^{k}\left[0, a_{1}\right]$ $=\left[0, b_{k}\right]$. Choose $l \geq 1$ such that $a_{l-1}<b_{k} \leq a_{l}$. Then

$$
T^{k+1}\left[0, a_{1}\right]=T_{1}\left[0, a_{1}\right] \cup \ldots \cup T_{l-1}\left[a_{l-2}, a_{l-1}\right] \cup T_{l}\left[a_{l-1}, b_{k}\right],
$$


where each of these sets is a closed interval. If the union is not connected, then there exists an interval $(c, d)$ with $d<\sup \left\{T(x) \mid x \in\left[0, b_{k}\right]\right\}$ and $\mathcal{F}_{l} \equiv 0$ on $(c, d)$ since the $F_{i}=0$ almost everywhere on this interval for $1 \leq i \leq l$ and the sum $\mathcal{F}_{l}$ is upper semicontinuous. But also, there exists a point $y>d$ such that $\mathcal{F}_{l}(y)>0$, which contradicts our convexity condition (C1). So $T^{k+1}\left[0, a_{1}\right]$ is an interval. Now note that since $T_{1}$ is convex and $F_{1}(0)<1$ we have $\left[0, a_{1}\right] \subseteq T_{1}\left[0, a_{1}\right]$, so $T^{k+1}\left[0, a_{1}\right] \supseteq\left[0, a_{1}\right]$. Finally, since $\left[0, a_{1}\right] \subseteq T\left[0, a_{1}\right]$, it follows that for all $k, T^{k}\left[0, a_{1}\right] \subseteq T^{k+1}\left[0, a_{1}\right]$.

Let $\beta \in(0,1]$ be determined by $\bigcup_{k=0}^{\infty} T^{k}\left[0, a_{1}\right]=[0, \beta]$. Then, by Lemma 4.1, $a_{1}<\beta \leq 1$ and $T[0, \beta]=[0, \beta]$. By Remark 2.2 , without loss of generality, we may assume that $\beta=a_{N_{*}}$ for some $N_{*} \in\{2, \ldots, N\}$. Then $\mathcal{F}_{N_{*}}(x)=0$ on $(\beta, 1]$ and for any $c \in(0, \beta), T[0, c] \nsubseteq[0, c]$, which leads to the following (see Figure 4.1).

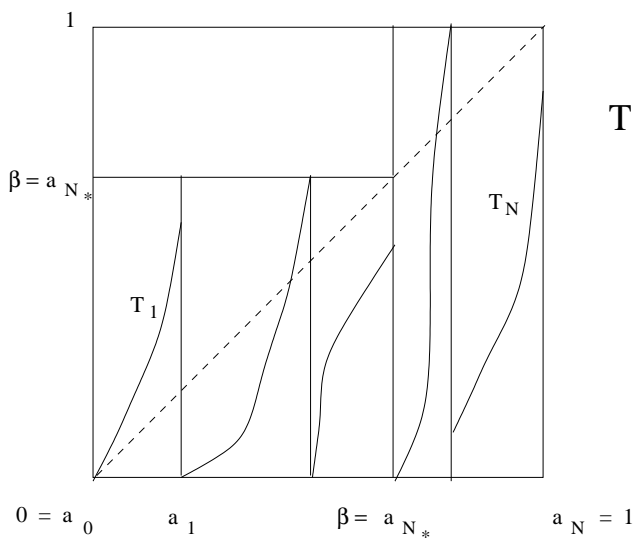

Fig. 4.1

Lemma 4.2. For each $c \in(0, \beta), T[c, \beta] \nsubseteq[c, \beta]$.

Proof. Suppose $0<c<\beta$ and $T[c, \beta] \subseteq[c, \beta]$. Again, in view of Remark 2.2 , without loss of generality, we may assume that $c=a_{s}$ for some $s, 1 \leq$ $s<N_{*}$. It follows that $\sum_{i=s+1}^{N_{*}} F_{i}(x)=0$ on $[0, c)$, i.e., $\left(\mathcal{F}_{N_{*}}-\mathcal{F}_{s}\right)(x)=0$ on $[0, c)$. Also

$$
c=\int_{0}^{1} P \chi_{[0, c]}(t) d t=\int_{0}^{1} \mathcal{F}_{s}(t) d t \geq \int_{0}^{c} \mathcal{F}_{s}(t) d t \geq c \mathcal{F}_{s}(c),
$$

which means $\mathcal{F}_{s}(c) \leq 1$. Since $\mathcal{F}_{s}(x)=\mathcal{F}_{N_{*}}(x)$ on $[0, c)$, this implies that $\lim _{x \rightarrow c^{-}} \mathcal{F}_{N_{*}}(x) \leq 1$, and so $\mathcal{F}_{N_{*}}(c) \leq 1$. Thus

$$
\int_{c}^{\beta} \mathcal{F}_{N_{*}}(t) d t \leq(\beta-c) \mathcal{F}_{N_{*}}(c) \leq \beta-c .
$$


Meanwhile,

$$
\begin{aligned}
\beta-c & =\int_{0}^{1} P \chi_{[c, \beta]}(t) d t=\int_{0}^{1}\left(\mathcal{F}_{N_{*}}-\mathcal{F}_{s}\right)(t) d t \\
& =\int_{c}^{\beta}\left(\mathcal{F}_{N_{*}}-\mathcal{F}_{s}\right)(t) d t \leq \int_{c}^{\beta} \mathcal{F}_{N_{*}}(t) d t .
\end{aligned}
$$

Therefore $\int_{c}^{\beta} \mathcal{F}_{N_{*}}(t) d t=\beta-c$ and $\int_{c}^{\beta} \mathcal{F}_{s}(t) d t=0$. Then $T[0, c] \subseteq[0, c]$, which is a contradiction.

LEMMA 4.3. There exists an integer $r \geq 1$ such that $\bigcup_{k=0}^{r} T^{k}\left[0, a_{1}\right]=$ $[0, \beta]$.

Proof. Let $d=\max \left\{T_{i}\left(a_{i}\right) \mid 1 \leq i \leq N_{*}-1\right\} \quad(\leq \beta)$. Then $d>a_{N_{*}-1}$. For, otherwise, $\bigcup_{k=0}^{\infty} T^{k}\left[0, a_{1}\right] \subseteq\left[0, a_{N_{*}-1}\right] \subsetneq[0, \beta]$, which is a contradiction. We claim $d=\beta$, in which case it is easy to see that there exists $r \geq 1$ such that $\bigcup_{k=0}^{r} T^{k}\left[0, a_{1}\right]=[0, \beta]$. To prove the claim, first suppose $T_{N_{*}}(d)>d$. Then $T[d, \beta] \subseteq[d, \beta]$ and so by Lemma $4.2, d=\beta$. If $T_{N_{*}}(d) \leq d$, then $T[0, d] \subseteq[0, d]$, which implies $d=\beta$.

LEMMA 4.4. Let $g \in \mathcal{J}$ be an invariant density for $T$ and $A=\int_{0}^{\beta} g d m$. Define $g_{\beta}:[0,1] \rightarrow \mathbb{R}^{+}$by

$$
g_{\beta}(x)= \begin{cases}g(x) / A & \text { if } 0 \leq x \leq \beta \\ 0 & \text { if } \beta<x \leq 1 .\end{cases}
$$

Then $g_{\beta} \in \mathcal{J}$ is an invariant density of $T$, i.e., $P g_{\beta}=g_{\beta}$.

Proof. Let $g_{1}=g \chi_{[0, \beta]}$ and $g_{2}=g-g_{1}$. Then $g_{1}+g_{2}=P g_{1}+P g_{2}$. Since $T[0, \beta] \subseteq[0, \beta]$, we have $g_{1} \geq P g_{1}$. From the fact that $\left\|P g_{1}\right\|=\left\|g_{1}\right\|$, it follows that $P g_{1}=g_{1}$. Thus $g_{\beta}=g_{1} /\left\|g_{1}\right\|_{1} \in \mathcal{J}$ is an invariant density of $T$.

REMARK 4.1. For the rest of this and the following section, we will study the ergodic properties of $\left(T, g_{\beta} d m\right)$. In effect we will study $T_{\beta}=\left.T\right|_{[0, \beta]}$, an $N_{*}$-branched convex map on $[0, \beta]$. The Perron-Frobenius operator $P_{\beta}$ on $L^{1}(\beta)=L^{1}([0, \beta])$ is defined according to $(1.2)$ using $T_{\beta}$. The connection between the two operators is

$$
P_{\beta}=\left.P\right|_{L^{1}(\beta)} .
$$

This is easily seen from the representation (1.2). Similarly, we will adopt the notation $\mathrm{BV}(\beta), \mathrm{BV}_{0}(\beta), \mathcal{J}(\beta)$ and $\mathcal{C}_{K}(\beta), K>0$, to denote the function spaces and cones on the restricted domain $[0, \beta]$. For example it easily follows from Lemma 3.3 that for a given $K \geq b /(1-a), P_{\beta}$ preserves $\mathcal{C}_{K}(\beta)$, i.e., $P_{\beta} \operatorname{maps} \mathcal{C}_{K}(\beta)$ into $\mathcal{C}_{K}(\beta)$. 
Recall that the condition (C2) implies only that $T_{\beta}$ is uniformly expanding on $\left[0, a_{1}\right]$, however, it need not be the case that $T_{\beta}$ (or even some power of it) must be uniformly expanding on $[0, \beta]$. Surprisingly, if in addition, $T_{\beta}$ is assumed to be expanding at $\beta$, then we will prove that some power of $T_{\beta}$ is uniformly expanding. This motivates the terminology in the following:

We say $T_{\beta}$ satisfies the expanding condition (E) if

$$
F_{N_{*}}\left(\beta^{-}\right)<1 \text {. }
$$

We say $(T, \mu)$ has exponential decay of correlations if there exist $C<\infty$ and $\lambda<1$ such that for any $h \in L^{1}(\mu), f \in \mathrm{BV}(\mu)$, and for each $k \geq 1$,

$$
\left|\int\left(h \circ T^{k}\right) f d \mu-\int h d \mu \int f d \mu\right| \leq C \lambda^{k}\|h\|_{1}\|f\|_{\mathrm{BV}} .
$$

We first show the following.

TheOREM 4.5. Suppose $T_{\beta}$ satisfies the conditions in Theorem 1.1 and the expanding condition (E). Let $g_{\beta}$ be an invariant density of $T_{\beta}$ as defined in Lemma 4.4. Then $\left(T_{\beta}, g_{\beta} d m\right)$ is exact. Moreover it has exponential decay of correlations.

The proof of Theorem 4.5 results from a series of lemmas which will be proved later. Similar methods may be found in the work of Bowen [1].

To simplify notation, and in view of the above correspondences, we will generally refrain from including the subscript $\beta$ in $T$ and $P$ with the underlying assumption in this and the next section that the domain has been restricted to $[0, \beta]$.

Lemma 4.6. Let $r \geq 1$ be given as in Lemma 4.3. Let $K>0$ be given and choose $s \geq 0$ so that $\psi_{1}^{s}\left(a_{1}\right)<1 / K$. Then:

(1) For each $x \leq a_{1}$, there is $\lambda_{0}=\lambda_{0}(x)>0$ such that for any $f \in \mathcal{C}_{K}$, $\left(P^{s} f\right)(x) \geq \lambda_{0}\|f\|$.

(2) For each $x<\beta$, there is $\delta_{0}=\delta_{0}(x)>0$ such that for any $f \in \mathcal{C}_{K}$, $\left(P^{r+s} f\right)(x) \geq \delta_{0}\|f\|$.

LemmA 4.7. Let $K>b /(1-a)$. Then there exist $l=l(K) \geq 1$ and $h \in \mathcal{J}(\beta)$ such that $\int h>0$ and for any $f \in \mathcal{C}_{K}(\beta), P^{l} f-\|f\| h \in \mathcal{J}(\beta)$.

LemMA 4.8. Let $K>b /(1-a)$. Then there exist $n=n(K) \geq 1$ and $\widehat{h} \in \mathcal{J}(\beta)$ such that $\int \widehat{h}>0$ and for any $f \in \mathcal{C}_{K}(\beta), P^{n} f-\|f\| \widehat{h} \in \overline{\mathcal{C}}_{K}(\beta)$.

Proposition 4.9. Let $K>b /(1-a)$. Then there exist $n=n(K) \geq 1$ and $\delta=\delta(K)>0$ such that for any given $f \in \mathrm{BV}_{0}(\beta)$, for each $k \geq 1$,

$$
\left\|P^{k n} f\right\|_{\Gamma_{K}} \leq(1-\delta)^{k}\|f\|_{\Gamma_{K}} .
$$

Proof of Theorem 4.5. Fix $K>b /(1-a)$. It follows from Proposition 4.9 that there exist $n=n(K) \geq 1$ and $\delta=\delta(K)>0$ such that for any given 
$f \in \mathrm{BV}_{0}(\beta)$, for each $k \geq 1$,

$$
\left\|P^{k n} f\right\|_{\Gamma_{K}} \leq(1-\delta)^{k}\|f\|_{\Gamma_{K}} .
$$

Since the right hand side converges to 0 as $k \rightarrow \infty$, the left hand side converges to 0 as $k \rightarrow \infty$ and so by Lemma 3.2(4) in $\|\cdot\|_{\mathrm{BV}}$, which implies

$$
\left\|P^{k n} f\right\| \rightarrow 0 \quad \text { as } k \rightarrow \infty \text {. }
$$

Since $P$ is a contraction in $\|\cdot\|$,

$$
\left\|P^{m} f\right\| \rightarrow 0 \quad \text { as } m \rightarrow \infty .
$$

This will be enough for the exactness of the a.c.i.m. (see, for example, [7] where the term asymptotic stability is used). In fact, let $\phi \in \mathrm{BV}(\beta)$ and $\phi \geq 0$. Noticing that $f=\phi-\left(\int \phi\right) g_{\beta} \in \mathrm{BV}_{0}(\beta)$, we have $\left\|P^{m} f\right\| \rightarrow 0$ as $m \rightarrow \infty$, i.e., $\lim _{m \rightarrow \infty} P^{m} \phi=\left(\int \phi\right) g_{\beta}$ in $L_{1}$. Therefore, $\left(T, g_{\beta} d m\right)$ is exact.

To prove the second statement, fix $K>b /(1-a)$. Notice that $P$ is also a contraction in $\|\cdot\|_{\Gamma_{K}}$. It follows from Lemma 3.2 and Proposition 4.9 that there exist $C_{1}=C_{1}(K)<\infty$ and $\lambda=\lambda(K)<1$ such that for any given $f \in \mathrm{BV}_{0}(\beta)$, for each $k \geq 1$,

$$
\left\|P^{k} f\right\|_{\mathrm{BV}} \leq C_{1} \lambda^{k}\|f\|_{\mathrm{BV}}
$$

Let $h \in L^{1}$ and $f \in \mathrm{BV}$. Then with $d \mu=g_{\beta} d m$, we have $\widehat{f}=f-\int f d \mu \in \mathrm{BV}$ and $\widehat{f} g_{\beta} \in \mathrm{BV}_{0}(\beta)$. Now, observe that $\|\widehat{f}\|_{\infty} \leq \mathrm{V} \widehat{f}=\mathrm{V} f$ and since $g_{\beta}$ is positive, decreasing and integrates to 1 , we have $\mathrm{V} g_{\beta} \leq\left\|g_{\beta}\right\|_{\infty}$. Put this together to estimate $\mathrm{V}\left(\widehat{f} g_{\beta}\right) \leq \mathrm{V} \widehat{f}\left\|g_{\beta}\right\|_{\infty}+\|\widehat{f}\|_{\infty} \mathrm{V} g_{\beta} \leq 2\left\|g_{\beta}\right\|_{\infty} \mathrm{V} f$. Similar reasoning leads to $\left\|g_{\beta}\right\|_{\infty} \geq 1$ so we have $\left\|\widehat{f} g_{\beta}\right\| \leq\left\|g_{\beta}\right\|_{\infty}\|\widehat{f}\| \leq$ $\left\|g_{\beta}\right\|_{\infty}\left(\|f\|+\left\|g_{\beta}\right\|_{\infty}\|f\|\right)=\left\|g_{\beta}\right\|_{\infty}\left(1+\left\|g_{\beta}\right\|_{\infty}\right)\|f\|$, and therefore $\left\|\widehat{f} g_{\beta}\right\|_{\mathrm{BV}} \leq$ $\left(1+\left\|g_{\beta}\right\|_{\infty}\right)\left\|g_{\beta}\right\|_{\infty}\|f\|_{\mathrm{BV}}$. Thus for each $k \geq 1$,

$$
\begin{gathered}
\left|\int\left(h \circ T^{k}\right) f d \mu-\int h d \mu \int f d \mu\right|=\left|\int\left(h \circ T^{k}\right) \widehat{f} d \mu\right|=\left|\int h P^{k}\left(\widehat{f} g_{\beta}\right) d m\right| \\
\leq\|h\| \cdot\left\|P^{k}\left(\widehat{f} g_{\beta}\right)\right\|_{\infty} \leq\|h\| \cdot\left\|P^{k}\left(\widehat{f} g_{\beta}\right)\right\|_{\mathrm{BV}} \leq\|h\| C_{1} \lambda^{k}\left\|\widehat{f} g_{\beta}\right\|_{\mathrm{BV}} \\
\leq\|\cdot\| C_{1} \lambda^{k}\left(1+\left\|g_{\beta}\right\|_{\infty}\right)\left\|g_{\beta}\right\|_{\infty}\|f\|_{\mathrm{BV}} \leq C \lambda^{k}\|h\| \cdot\|f\|_{\mathrm{BV}}
\end{gathered}
$$

where $C=C_{1}\left(1+\left\|g_{\beta}\right\|_{\infty}\right)\left\|g_{\beta}\right\|_{\infty}<\infty$. Therefore $\left(T, g_{\beta} d m\right)$ has exponential decay of correlations.

Now in order to prove Lemma 4.6, we will use the following notations. For each $n \geq 1,\left\{a_{i}^{(n)}\right\}_{i=0}^{N_{n}}$ denotes the partition for $T^{n}$; for each $i=1, \ldots, N_{n}$, we define $T_{i}^{(n)}, \psi_{i}^{(n)}$, and $F_{i}^{(n)}$ similarly.

Proof of Lemma 4.6. (1) Let $y<1 / K$. We will first show that there exists $\varepsilon=\varepsilon(y)>0$ such that for any $f \in \mathcal{C}_{K}$,

$$
f(y) \geq \varepsilon\|f\| \text {. }
$$


In fact, define $\varepsilon=\varepsilon(y)$ by

$$
\varepsilon=\frac{1-K y}{1+K(1-y)}>0 .
$$

Then for a given $f \in \mathcal{C}_{K}$,

$$
f(0)-f(y) \leq f(0)-f(1)=\mathrm{V} f \leq K\|f\| \leq K[f(0) y+f(y)(1-y)],
$$

which leads to

$$
[1+K(1-y)] f(y) \geq(1-K y) f(0) \geq(1-K y)\|f\|,
$$

i.e., $f(y) \geq \varepsilon\|f\|$.

Let $x \leq a_{1}$ be given. Then $F_{1}^{(s)}(x)>0$ and $\psi_{1}^{(s)}(x) \leq \psi_{1}^{(s)}\left(a_{1}\right)<1 / K$. Let $\lambda_{0}=\lambda_{0}(x)=F_{1}^{(s)}(x) \varepsilon\left(\psi_{1}^{(s)}(x)\right)>0$. It follows from (4.1) that for any $f \in \mathcal{C}_{K}$,

$$
\begin{aligned}
\left(P^{s} f\right)(x) & =\sum_{i=1}^{N_{s}} F_{i}^{(s)}(x) f\left(\psi_{i}^{(s)}(x)\right) \geq F_{1}^{(s)}(x) f\left(\psi_{1}^{(s)}(x)\right) \\
& \geq F_{1}^{(s)}(x) \varepsilon\left(\psi_{1}^{(s)}(x)\right)\|f\|=\lambda_{0}\|f\| .
\end{aligned}
$$

(2) Note that for any $x<\beta, F_{1}^{(r)}(x)>0$. Fix $x<\beta$. It follows from (1) that $\lambda_{0}=\lambda_{0}\left(\psi_{1}^{(r)}(x)\right)>0$ is well defined, since $\psi_{1}^{(r)}(x) \leq a_{1}$. Let $\delta_{0}=$ $\delta_{0}(x)=F_{1}^{(r)}(x) \lambda_{0}\left(\psi_{1}^{(r)}(x)\right)>0$. From (1), for any given $f \in \mathcal{C}_{K}$,

$$
\begin{aligned}
\left(P^{r+s} f\right)(x) & =\sum_{i=1}^{N_{r}} F_{i}^{(r)}(x)\left(P^{s} f\right)\left(\psi_{i}^{(r)}(x)\right) \geq F_{1}^{(r)}(x)\left(P^{s} f\right)\left(\psi_{1}^{(r)}(x)\right) \\
& \geq F_{1}^{(r)}(x) \lambda_{0}\left(\psi_{1}^{(r)}(x)\right)\|f\|=\delta_{0}\|f\|,
\end{aligned}
$$

Proof of Lemma 4.7. Given a function $\phi$ on $I$, for each $x \in(0,1), \phi\left(x^{-}\right)$ will denote $\lim _{t \rightarrow x^{-}} \phi(t)$ provided the limit exists; similarly for $\phi\left(x^{+}\right)$. Let $r, s$ be given as in Lemma 4.6 and let $l=1+r+s \geq 2$.

The first guess for a choice of $h$ would be $h=\|f\| P \chi_{[0, \beta]}$ for then $h \in \mathcal{J}$ and $P^{l} f-h=P\left(P^{l-1} f-\|f\| \chi_{[0, \beta]}\right)$, which is decreasing on $[0, \beta]$. However, it is not the case that we can always choose $l$ large enough, independent of $f$ such that this function is positive on $[0, \beta]$. A slight modification is required.

We break the analysis into three cases.

CASE $1: d \equiv T\left(\beta^{-}\right)<\beta$. By Lemma 4.6(2), $\delta_{0}=\delta_{0}\left(a_{N_{*}-1}\right)>0$ is well defined. Noticing that $\left(P \chi_{[0, \beta]}\right)\left(d^{+}\right)=\mathcal{F}_{N_{*}-1}\left(d^{+}\right)>0$, let $t=$ $\delta_{0}\left(P \chi_{[0, \beta]}\right)\left(d^{+}\right)>0$ and define $h \in \mathcal{J}(\beta)$ by

$$
h=t \chi_{[0, d]}+\delta_{0} P \chi_{[0, \beta]} \cdot \chi_{(d, \beta]} .
$$


Then $\int h>0$. For a given $f \in \mathcal{C}_{K}(\beta)$, let $\widehat{f}=P^{r+s} f$ and observe

$$
(P \widehat{f}-\|f\| h)(x)= \begin{cases}(P \widehat{f})(x)-t\|f\| & \text { if } 0 \leq x \leq d, \\ (P \widehat{f})(x)-\delta_{0}\|f\|\left(P \chi_{[0, \beta]}\right)(x) & \text { if } d<x \leq \beta \\ 0 & \text { if } \beta<x \leq 1\end{cases}
$$

It is clear that $P \widehat{f}-\|f\| h$ is decreasing on $[0, d]$. Let $d<x \leq \beta$. For each $i=1, \ldots, N_{*}-1$,

$$
\widehat{f}\left(\psi_{i}(x)\right) \geq \widehat{f}\left(\psi_{N_{*}-1}(x)\right) \geq \widehat{f}\left(a_{N_{*}-1}\right) \geq \delta_{0}\|f\| .
$$

Thus

$$
(P \widehat{f}-\|f\| h)(x)=\sum_{i=1}^{N_{*}-1} F_{i}(x)\left[\widehat{f}\left(\psi_{i}(x)\right)-\delta_{0}\|f\|\right] \geq 0
$$

and it is decreasing on $(d, \beta]$. Using the fact that $h$ is continuous at $d$, we conclude that $P^{l} f-\|f\| h \geq 0$ is decreasing on $[0,1]$, i.e., $P^{l} f-\|f\| h \in \mathcal{J}(\beta)$.

Case 2: $T\left(\beta^{-}\right)=\beta$ and $P \chi_{[0, \beta]} \neq \chi_{[0, \beta]}$. Notice that there exist $c, d$ with $c<d<\beta$ such that $\left(P \chi_{[0, \beta]}\right)\left(c^{+}\right)>\left(P \chi_{[0, \beta]}\right)\left(d^{-}\right)$. Since $\psi_{N_{*}}\left(d^{+}\right)<\beta$, it follows from Lemma 4.6(2) that $\delta_{0}=\delta_{0}\left(\psi_{N_{*}}\left(d^{+}\right)\right)>0$ is well defined. Let

$$
t=\delta_{0}\left[\left(P \chi_{[0, \beta]}\right)\left(c^{+}\right)-\left(P \chi_{[0, \beta]}\right)\left(d^{-}\right)\right]>0
$$

and define $h \in \mathcal{J}(\beta)$ by

$$
h=t \chi_{[0, c]}+\delta_{0}\left[P \chi_{[0, \beta]}-\left(P \chi_{[0, \beta]}\right)\left(d^{-}\right)\right] \chi_{(c, d]} .
$$

Then $\int h>0$. Let $f \in \mathcal{C}_{K}(\beta)$ and $\widehat{f}=P^{r+s} f$. Letting $s=\delta_{0} P \chi_{[0, \beta]}\left(d^{-}\right)\|f\|$, we have

$$
(P \widehat{f}-\|f\| h)(x)= \begin{cases}(P \widehat{f})(x)-t\|f\| & \text { if } 0 \leq x \leq c \\ (P \widehat{f})(x)-\delta_{0}\|f\|\left(P \chi_{[0, \beta]}\right)(x)+s & \text { if } c<x \leq d \\ (P \widehat{f})(x) & \text { if } d<x \leq 1\end{cases}
$$

Observe that $P \widehat{f}-\|f\| h$ is decreasing on each of the three intervals $[0, c]$, $(c, d]$ and $(d, 1]$. Since $h$ is continuous at $c$ and $d$, we see that $P \widehat{f}-\|f\| h$ is decreasing on $[0,1]$. Since $(P \widehat{f}-\|f\| h)(1)=(P \widehat{f})(1) \geq 0$, it follows that $P^{l} f-\|f\| h \in \mathcal{J}(\beta)$.

CAse 3: $T\left(\beta^{-}\right)=\beta$ and $P \chi_{[0, \beta]}=\chi_{[0, \beta]}\left(\right.$ and $\left.F_{N_{*}}(\beta)<1\right)$. Since $a_{N_{*}-1}<\beta$, it follows from Lemma 4.6(2) that $\delta_{0}=\delta_{0}\left(a_{N_{*}-1}\right)>0$ is well defined. Let $t=\left(1-F_{N_{*}}(\beta)\right) \delta_{0}>0$ and define $h \in \mathcal{J}(\beta)$ by $h=t \chi_{[0, \beta]}$. Then $\int h>0$. We show that for a given $f \in \mathcal{C}_{K}(\beta), P^{l} f-\|f\| h \in \mathcal{J}(\beta)$. Let $\widehat{f}=P^{r+s} f$. It is clear that $P \widehat{f}-\|f\| h$ is decreasing. Using the fact that 
$\mathcal{F}_{N_{*}}(\beta)=1$, we have

$$
\begin{aligned}
(P \widehat{f})(\beta) & =\sum_{i=1}^{N_{*}} F_{i}(\beta) \widehat{f}\left(\psi_{i}(\beta)\right) \geq \sum_{i=1}^{N_{*}-1} F_{i}(\beta) \widehat{f}\left(\psi_{N_{*}-1}(\beta)\right) \\
& \geq \sum_{i=1}^{N_{*}-1} F_{i}(\beta) \widehat{f}\left(a_{N_{*}-1}\right) \geq\left(1-F_{N_{*}}(\beta)\right) \delta_{0}\|f\|=t\|f\| .
\end{aligned}
$$

Thus $P^{l} f-\|f\| h \in \mathcal{J}(\beta)$.

Proof of Lemma 4.8. Let $l=l(K) \geq 1$ and $h \in \mathcal{J}(\beta)$ be given as in Lemma 4.7. Choose $\delta>0$ so that

$$
0<\delta<\frac{K-b /(1-a)}{K+b /(1-a)}<1 .
$$

Note that $K>b(1+\delta) /[(1-\delta)(1-a)]>b(1+\delta) /(1-a)$. Let $\varepsilon=\mathrm{Vh} \geq 0$ and $\lambda=\min \left\{\delta / \int h, 1\right\}>0$. Since $a<1$ one may choose $m \geq 0$ so that

$$
m \geq \log _{a}\left[\frac{K(1-\delta)(1-a)-b(1+\delta)}{(K+\lambda \varepsilon)(1-a)-b(1+\delta)}\right] .
$$

A simple computation shows

$$
0<\left(K+\lambda \varepsilon-\frac{b(1+\delta)}{1-a}\right) a^{m}+\frac{b(1+\delta)}{1-a} \leq K(1-\delta) .
$$

Let $n=m+l \geq 1$ and $\widehat{h}=P^{m}(\lambda h) \in \mathcal{J}(\beta)$. For a given $f \in \mathcal{C}_{K}(\beta)$, let $\zeta=P^{n} f-\|f\| \widehat{h}$ and $\phi=\lambda\|f\| h \in \mathcal{J}$. Note that

$$
\zeta=P^{m}\left(P^{l} f-\lambda\|f\| h\right)=P^{m}\left(P^{l} f-\|f\| h\right)+(1-\lambda) P^{m}(\|f\| h)
$$

from which $\zeta \in \mathcal{J}$, since $P^{l} f-\|f\| h \in \mathcal{J}$ and $\|f\| h \in \mathcal{J}$. Also $\|\phi\| \leq \delta\|f\|$. Thus

$$
\|\zeta\|=\left\|P^{m}\left(P^{l} f-\lambda\|f\| h\right)\right\|=\left\|P^{n} f\right\|-\left\|P^{m} \phi\right\|=\|f\|-\|\phi\| \geq(1-\delta)\|f\| .
$$

Using (3.3) and (4.2), observe that

$$
\begin{aligned}
\mathrm{V} \zeta & \leq \mathrm{V} P^{m}\left(P^{l} f\right)+\mathrm{VP}^{m} \phi \\
& \leq\left(a^{m} \mathrm{~V}^{l} f+\frac{b\left(1-a^{m}\right)}{1-a}\left\|P^{l} f\right\|\right)+\left(a^{m} \mathrm{~V} \phi+\frac{b\left(1-a^{m}\right)}{1-a}\|\phi\|\right) \\
& \leq\left[a^{m} K+\frac{b\left(1-a^{m}\right)}{1-a}+a^{m} \lambda \varepsilon+\frac{b\left(1-a^{m}\right)}{1-a} \delta\right]\|f\| \\
& =\left[\left(K+\lambda \varepsilon-\frac{b(1+\delta)}{1-a}\right) a^{m}+\frac{b(1+\delta)}{1-a}\right]\|f\| \\
& \leq K(1-\delta)\|f\| \leq K\|\zeta\| .
\end{aligned}
$$

Clearly $\zeta(x)=0$ on $(\beta, 1]$. Therefore, $\zeta=P^{n} f-\|f\| \widehat{h} \in \mathcal{C}_{K}(\beta)$. 
Proof of Proposition 4.9. Fix $K>b /(1-a)$. Let $n=n(K) \geq 1$ and $\widehat{h} \in \mathcal{J}(\beta)$ be given as in Lemma 4.8. Let $\delta=\int \widehat{h}>0$. Suppose $f=f^{1}-f^{2} \in$ $\mathrm{BV}_{0}(\beta)$, where $f^{1}, f^{2} \in \mathcal{C}_{K}(\beta)$ and $\|f\|_{\Gamma_{K}}=\left\|f^{1}\right\|=\left\|f^{2}\right\|$ (such $f^{1}, f^{2}$ exist from Lemma 3.1). It follows from Lemma 4.8 that $P^{n} f^{1}-\left\|f^{1}\right\| \widehat{h} \in \mathcal{C}_{K}(\beta)$ and $P^{n} f^{2}-\left\|f^{2}\right\| \widehat{h} \in \mathcal{C}_{K}(\beta)$. Note that $P^{n} f=\left(P^{n} f^{1}-\left\|f^{1}\right\| \widehat{h}\right)-\left(P^{n} f^{2}-\left\|f^{2}\right\| \widehat{h}\right)$ and $\left\|P^{n} f^{1}-\right\| f^{1}\|\cdot \widehat{h}\|=\left\|P^{n} f^{2}-\right\| f^{2}\|\cdot \widehat{h}\|$. Thus

$$
\left\|P^{n} f\right\|_{\Gamma_{K}} \leq\left\|P^{n} f^{1}-\right\| f^{1}\|\cdot \widehat{h}\|=(1-\delta)\left\|f^{1}\right\|=(1-\delta)\|f\|_{\Gamma_{K}} .
$$

Since $P^{n} f \in \mathrm{BV}_{0}(\beta)$, it follows that

$$
\left\|P^{n}\left(P^{n} f\right)\right\|_{\Gamma_{K}} \leq(1-\delta)\left\|P^{n} f\right\|_{\Gamma_{K}} \leq(1-\delta)^{2}\|f\|_{\Gamma_{K}} .
$$

Repeating this process, we conclude that for each $k \geq 1,\left\|P^{k n} f\right\|_{\Gamma_{K}} \leq$ $(1-\delta)^{k}\|f\|_{\Gamma_{K}}$.

We will show some other ergodic properties of $\left(T, g_{\beta} d m\right)$ and use a notation such as $i_{1} \ldots i_{n}, n \geq 1$, to denote an index with $i_{k} \in\{1, \ldots, N\}$ for each $k=1, \ldots, n$. This notation is particularly involved in the map $T^{n}$; for a given index $i_{1} \ldots i_{n}, I_{i_{1} \ldots i_{n}}^{(n)}$ will denote the interval that is the domain of $T_{i_{n}} \circ \ldots \circ T_{i_{1}}$, and define

$$
F_{i_{1} \ldots i_{n}}^{(n)}=F_{i_{1}}\left(\psi_{i_{2}} \circ \ldots \circ \psi_{i_{n}}\right) F_{i_{2}}\left(\psi_{i_{3}} \circ \ldots \circ \psi_{i_{n}}\right) \ldots F_{i_{n-1}}\left(\psi_{i_{n}}\right) F_{i_{n}} .
$$

Then for almost every $x$,

$$
F_{i_{1} \ldots i_{n}}^{(n)}(x)=\left(\psi_{i_{1} \ldots i_{n}}^{(n)}\right)^{\prime}(x)=\left(\psi_{i_{1}} \circ \ldots \circ \psi_{i_{n}}\right)^{\prime}(x),
$$

so the $F_{i_{1} \ldots i_{n}}^{(n)}$ form a consistent set of weights for the Perron-Frobenius operator for $T^{n}$.

Let $\mathcal{I}$ denote the set of all finite strings of indices such as $i_{1} \ldots i_{n}$ with $i_{k} \in\{1, \ldots, N\}$ for each $k=1, \ldots, n$, and $\mathcal{I}_{\beta}=\left\{i_{1} \ldots i_{n} \in \mathcal{I} \mid 1 \leq i_{k} \leq N_{*}\right.$ for $k=1, \ldots, n\}$. Recall that $\beta=a_{N_{*}}$.

TheOREM 4.10. Suppose $T$ satisfies the conditions in Theorem 1.1 and the expanding condition (E). Then

$$
\limsup _{n \rightarrow \infty} \max _{i_{1} \ldots i_{n} \in \mathcal{I}_{\beta}}\left\|F_{i_{1} \ldots i_{n}}^{(n)}\right\|_{\infty}<1
$$

Corollary 4.11. If $T$ satisfies the conditions in Theorem 1.1 and the expanding condition $(\mathrm{E})$, then the partition of $I=[0, \beta]$ into monotonicity intervals for $T$ is weak-Bernoulli for $\left(T_{\beta}, g_{\beta} d x\right)$. The natural extension of this system is therefore isomorphic to a Bernoulli shift.

Proof. One can easily deduce that

$$
\lim _{n \rightarrow \infty} \max _{i_{1} \ldots i_{n} \in \mathcal{I}_{\beta}}\left\|F_{i_{1} \ldots i_{n}}^{(n)}\right\|_{\infty}^{1 / n}<1
$$


Choose a power $s>0$ such that $T_{\beta}^{s}$ is uniformly expanding on $[0, \beta]$. Combining this with the exponential rate of decay in Theorem 4.5 (applied to $T_{\beta}^{s}$ ), it is not difficult to show directly that the monotonicity partition for $T_{\beta}^{s}$ is weak-Bernoulli, and by elementary argument, so is the original monotonicity partition for $T_{\beta}$. Alternatively, the article of Rychlik [11] may be invoked. A few comments are in order. First the assumptions in [11] may appear to be incompatible with our convexity assumption (C), however, note that the latter implies the weight function $g$ (in the notation of [11]) is of bounded variation. The condition that $\left.g\right|_{S} \equiv 0$ is not generally satisfied by our maps, but can be obtained with a measure-zero perturbation of our weight function, so the operator $P$ in that work is identified with our Perron-Frobenius operator (1.2). The proof of the weak-Bernoulli property in $\S 3$ of [11] depends only on the uniform expanding condition and the fact that the peripheral spectrum of the operator $P$ consists of one simple eigenvalue at 1 . These follow from our Theorem 4.5 applied to $T_{\beta}^{s}$.

REMARK 4.2. The convexity condition (C) always guarantees that

$$
\max _{i_{1} \ldots i_{n} \in \mathcal{I}_{\beta}}\left\|F_{i_{1} \ldots i_{n}}^{(n)}\right\|_{\infty} \leq 1
$$

for all $n$ large enough. This is obvious from Theorem 4.10 when $F_{N_{*}}(\beta)<1$, i.e., $T$ satisfies the expanding condition (E). Suppose $F_{N_{*}}(\beta)=1$. It follows that $P \chi_{[0, \beta]}=\chi_{[0, \beta]}$, or equivalently, $\mathcal{F}_{N_{*}}=\chi_{[0, \beta]}$, which means that for each $j=1, \ldots, N_{*}, F_{j}(x) \leq 1$ for all $x \in[0,1]$. Thus for all $n \geq 1$,

$$
\max _{i_{1} \ldots i_{n} \in \mathcal{I}_{\beta}}\left\|F_{i_{1} \ldots i_{n}}^{(n)}\right\|_{\infty} \leq 1
$$

In fact, in case $F_{N_{*}}(\beta)=1$, we have $T_{N_{*}}(\beta)=\beta$, so that $\psi_{N_{*}}(\beta)=\beta$. Thus for each $n \geq 1$,

$$
\max _{i_{1} \ldots i_{n} \in \mathcal{I}_{\beta}}\left\|F_{i_{1} \ldots i_{n}}^{(n)}\right\|_{\infty} \geq F_{N_{*} \ldots N_{*}}^{(n)}(\beta)=\prod_{k=0}^{n-1} F_{N_{*}}\left(\psi_{N_{*}}^{k}(\beta)\right)=\prod_{k=0}^{n-1} F_{N_{*}}(\beta)=1,
$$

which implies

$$
\max _{i_{1} \ldots i_{n} \in \mathcal{I}_{\beta}}\left\|F_{i_{1} \ldots i_{n}}^{(n)}\right\|_{\infty}=1
$$

Thus $F_{N_{*}}(\beta)<1$ if and only if some power of $T$ is expanding on $[0, \beta]$ (see [5]).

To prove Theorem 4.10, we first present two simple observations which require the convexity only.

Lemma 4.12. There exists $M \geq 1$ such that for all $m \geq 1,\left\|P^{m} \mathbf{1}\right\|_{\infty} \leq M$. 
Proof. Recall (the paragraph preceding Lemma 3.3) that there exist $a \in$ $\left(F_{1}(0), 1\right)$ and $b<\infty$ such that for any given $f \in \mathcal{J}$ and for any $m \geq 1$,

$$
\mathrm{V} P^{m} f \leq a^{m} \mathrm{~V} f+\frac{b\left(1-a^{m}\right)}{1-a}\|f\| .
$$

With $f=\mathbf{1} \in \mathcal{J}$, we have $\mathrm{V} f=0$ and $\|f\|=1$. Thus for any $m \geq 1$,

$$
\mathrm{V} P^{m} \mathbf{1} \leq \frac{b}{1-a} .
$$

Since $\left(P^{m} \mathbf{1}\right)(1) \leq 1$, it follows that

$$
\mathrm{V} P^{m} \mathbf{1}=\left(P^{m} \mathbf{1}\right)(0)-\left(P^{m} \mathbf{1}\right)(1) \geq\left(P^{m} \mathbf{1}\right)(0)-1,
$$

so that

$$
\left\|P^{m} \mathbf{1}\right\|_{\infty}=\left(P^{m} \mathbf{1}\right)(0) \leq \mathrm{V} P^{m} \mathbf{1}+1 \leq \frac{b}{1-a}+1 .
$$

Letting $M=b /(1-a)+1$ completes the proof.

LEMMA 4.13. Let $n \geq 1$ and $F_{i_{1} \ldots i_{n}}^{(n)}\left(x^{*}\right) \geq B>0$ for some index $i_{1} \ldots i_{n}$ and $x^{*} \in[0,1]$. Then for any given $p, q, 0 \leq p<q \leq n$, letting $x_{q+1}=$ $\psi_{i_{q+1}} \circ \psi_{i_{q+2}} \circ \ldots \circ \psi_{i_{n}}\left(x^{*}\right)\left(x_{n+1}=x^{*}\right)$, we have

$$
F_{i_{p+1} \ldots i_{q}}^{(q-p)}\left(x_{q+1}\right) \geq B / M^{2} .
$$

Proof. Let $x_{p+1}=\psi_{i_{p+1}} \circ \psi_{i_{p+2}} \circ \ldots \circ \psi_{i_{n}}\left(x^{*}\right)$. If $1 \leq p<q \leq n-1$, then it immediately follows from Lemma 4.12 that

$$
\begin{aligned}
B & \leq F_{i_{1} \ldots i_{n}}^{(n)}\left(x^{*}\right)=F_{i_{1} \ldots i_{p}}^{(p)}\left(x_{p+1}\right) F_{i_{p+1} \ldots i_{q}}^{(q-p)}\left(x_{q+1}\right) F_{i_{q+1} \ldots i_{n}}^{(n-q)}\left(x^{*}\right) \\
& \leq M F_{i_{p+1} \ldots i_{q}}^{(q-p)}\left(x_{q+1}\right) M
\end{aligned}
$$

which implies $F_{i_{p+1} \ldots i_{q}}^{(q-p)}\left(x_{q+1}\right) \geq B / M^{2}$.

If $p=0$ or $q=n$, then similarly we have

$$
B \leq F_{i_{1} \ldots i_{n}}^{(n)}\left(x^{*}\right) \leq M F_{i_{p+1} \ldots i_{q}}^{(q-p)}\left(x_{q+1}\right)
$$

which implies that $F_{i_{p+1} \ldots i_{q}}^{(q-p)}\left(x_{q+1}\right) \geq B / M \geq B / M^{2}$.

Lemma 4.14. Suppose the assumption in Theorem 4.10 holds. Then for each $j=1, \ldots, N_{*}$, there exists $\alpha(j) \in \mathbb{N}$ such that

$$
\left\|\prod_{k=1}^{\alpha(j)} F_{j}\left(\psi_{j}^{k-1}\right)\right\|_{\infty}<\frac{1}{2 M^{2}} .
$$

Proof. For $j=1$, since $\left\|F_{1}\right\|_{\infty}<1$, there exists $m \geq 1$ such that $\left(\left\|F_{1}\right\|_{\infty}\right)^{m}<1 /\left(2 M^{2}\right)$. With $\alpha(1)=m$, it is clear that

$$
\left\|\prod_{k=1}^{\alpha(1)} F_{1}\left(\psi_{1}^{k-1}\right)\right\|_{\infty} \leq\left(\left\|F_{1}\right\|_{\infty}\right)^{\alpha(1)}<\frac{1}{2 M^{2}} .
$$


Fix $j, 2 \leq j \leq N$. First suppose either $T_{j}(x)<x$ for all $x \in I_{j}=$ $\left[a_{j-1}, a_{j}\right]$, or that $T_{j}(x)>x$ for all $x \in I_{j}$. Then either $T_{j}\left(a_{j}\right)<a_{j}$ or $T_{j}\left(a_{j-1}\right)>a_{j-1}$. It is easy to see that there exists $m \geq 1$ such that either for any $k \geq m$,

$$
\psi_{j}^{k}(x)>\frac{a_{j}+T_{j}\left(a_{j}\right)}{2}>T_{j}\left(a_{j}\right) \quad \text { for all } x,
$$

or for any $k \geq m$,

$$
\psi_{j}^{k}(x)<\frac{a_{j-1}+T_{j}\left(a_{j-1}\right)}{2}<T_{j}\left(a_{j-1}\right) \quad \text { for all } x,
$$

which implies in either case that for any $k \geq m$, we have $F_{j}\left(\psi_{j}^{k}(x)\right)=0$ for all $x$. Letting $\alpha(j)=m+1$, we get

$$
\left\|\prod_{k=1}^{\alpha(j)} F_{j}\left(\psi_{j}^{k-1}\right)\right\|_{\infty}=0
$$

which completes the proof in this case.

Now assume $2 \leq j \leq N_{*}$ and $T_{j}\left(z^{*}\right)=z^{*}\left(\right.$ so $\psi_{j}\left(z^{*}\right)=z^{*}$ ) for some $z^{*} \in\left[a_{j-1}, a_{j}\right]\left(0<z^{*} \leq \beta\right.$ if such $z^{*}$ exists $)$. Then

$$
\begin{aligned}
z^{*} & =\psi_{j}\left(z^{*}\right)=\int_{0}^{z^{*}} F_{j}(x) d x+a_{j-1}=\int_{0}^{z^{*}} \mathcal{F}_{j}(x) d x+\int_{z^{*}}^{1} \mathcal{F}_{j-1}(x) d x \\
& \geq \int_{0}^{z^{*}} \mathcal{F}_{j}(x) d x \geq z^{*} \mathcal{F}_{j}\left(z^{*}\right)
\end{aligned}
$$

since $\mathcal{F}_{j}=\sum_{i=1}^{j} F_{i}$ is decreasing. Thus $\mathcal{F}_{j}\left(z^{*}\right) \leq 1$ and hence for any $x \geq z^{*}$, $\mathcal{F}_{j}(x) \leq 1$.

We claim that such a $z^{*}$ is unique. In fact, if $\psi_{j}\left(z_{0}\right)=z_{0}, \psi_{j}\left(z_{1}\right)=z_{1}$, and $0<z_{0}<z_{1} \leq \beta$, then $F_{j}(x) \leq 1$ on $\left[z_{0}, 1\right]$ so that $z_{1}-z_{0}=\int_{z_{0}}^{z_{1}} F_{j}(t) d t$ and hence $F_{j}(x)=1$ on $\left[z_{0}, z_{1}\right]$. Then $\mathcal{F}_{j-1}(x)=0$ on $\left[z_{0}, z_{1}\right]$. Thus $\beta \leq z_{0}$, which is a contradiction.

We will show that there exist $\varepsilon>0$ and $w^{*}<z^{*}$ such that for any $y \in\left[w^{*}, 1\right], F_{j}(y) \leq 1-\varepsilon<1$. First suppose $\mathcal{F}_{j}\left(z^{*}\right)<1$. The assumption that $\mathcal{F}_{j}$ is upper semicontinuous implies then that there exist $\varepsilon>0$ and $w^{*}<z^{*}$ such that for any $y \in\left[w^{*}, 1\right], \mathcal{F}_{j}(y) \leq 1-\varepsilon$ and so $F_{j}(y) \leq 1-\varepsilon<1$. Next in case $\mathcal{F}_{j}\left(z^{*}\right)=1$, it follows from $(4.3)$ that $\mathcal{F}_{j}(x)=1$ on $\left[0, z^{*}\right]$ and $\mathcal{F}_{j-1}(x)=0$ on $\left(z^{*}, 1\right]$. Thus $\beta \leq z^{*}$, which means $z^{*}=\beta$ and so $j=N_{*}$. Then $\mathcal{F}_{N_{*}}(x)=1$ on $[0, \beta]$, which indicates that $F_{N_{*}}$ is increasing on $[0, \beta]$. Since $F_{N_{*}}(\beta)<1$, it easily follows that there exists $\varepsilon\left(=1-F_{N_{*}}(\beta)\right)>0$ such that for any $y \in[0,1], F_{j}(y)=F_{N_{*}}(y) \leq 1-\varepsilon<1$. Therefore in either case, there exist $\varepsilon>0$ and $w^{*}<z^{*}$ such that for any $y \in\left[w^{*}, 1\right], F_{j}(y) \leq 1-\varepsilon<1$.

Note that for any $x<z^{*}, \psi_{j}^{k}(x) \nearrow z^{*}$ as $k \rightarrow \infty$ and for any $x>z^{*}$, $\psi_{j}^{k}(x) \searrow z^{*}$ as $k \rightarrow \infty$. Thus there exists $m \geq 1$ such that for any given 
$k \geq m$, for any $x \in[0,1], \psi_{j}^{k}(x)>w^{*}$ and hence $F_{j}\left(\psi_{j}^{k}(x)\right) \leq 1-\varepsilon$. Let

$$
\alpha(j)=\left[m-\frac{\ln \left(2 M^{3}\right)}{\ln (1-\varepsilon)}\right]+1 \text {. }
$$

By Lemma 4.12,

$$
\begin{aligned}
\left\|\prod_{k=1}^{\alpha(j)} F_{j}\left(\psi_{j}^{k-1}\right)\right\|_{\infty} & \leq\left\|\prod_{k=1}^{m} F_{j}\left(\psi_{j}^{k-1}\right)\right\|_{\infty}\left\|\prod_{k=m+1}^{\alpha(j)} F_{j}\left(\psi_{j}^{k-1}\right)\right\|_{\infty} \\
& \leq\|F_{m}^{(m)} \underbrace{(m)}_{m j}\|_{\infty} \prod_{k=m+1}^{\alpha(j)}\left\|F_{j}\left(\psi_{j}^{k-1}\right)\right\|_{\infty} \\
& \leq M(1-\varepsilon)^{\alpha(j)-m}<\frac{1}{2 M^{2}} .
\end{aligned}
$$

We now introduce some notations and definitions.

Definition 4.1. (1) For $n \geq 3$, an index $i_{1} \ldots i_{n}$ is said to be bowlshaped if there exists $r, 2 \leq r \leq n-1$, for which $i_{1}>i_{r}$ and

$$
i_{1} \geq \ldots \geq i_{r}<i_{r+1}<\ldots<i_{n} .
$$

(2) For $n \geq 1$, an index $i_{1} \ldots i_{n}$ is said to be increasing if $i_{1} \leq \ldots \leq i_{n}$.

(3) For $n \geq 1$, an index $i_{1} \ldots i_{n}$ is said to be decreasing if $i_{1} \geq \ldots \geq i_{n}$.

(4) For $n \geq 1$, an index $i_{1} \ldots i_{n}$ is said to be monotone if it is either increasing or decreasing.

Under the notations in Lemma 4.14, let $\alpha=\sum_{j=1}^{N_{*}} \alpha(j)$.

Lemma 4.15. Suppose the assumption in Theorem 4.10 holds. Let $n \geq 1$ and an index $i_{1} \ldots i_{n} \in \mathcal{I}_{\beta}$ be given so that $\left\|F_{i_{1} \ldots i_{n}}^{(n)}\right\|_{\infty} \geq 1$. If for some $s, t$, $1 \leq s<t \leq n$, a subindex $i_{s} \ldots i_{t}$ is monotone, then $t-s<\alpha$.

Proof. Suppose $t-s \geq \alpha$. Since either $i_{s} \leq \ldots \leq i_{t}$ or $i_{s} \geq \ldots \geq i_{t}$, there exist $j$ and $r$ such that $1 \leq j \leq N_{*}$ and $s-1 \leq r \leq t-\alpha(j)$ and

$$
i_{r+1}=\ldots=i_{r+\alpha(j)}=j .
$$

Using Lemma 4.12 and Lemma 4.14, we obtain

$$
\begin{aligned}
1 & \leq\left\|F_{i_{1} \ldots i_{n}}^{(n)}\right\|_{\infty} \leq\left\|F_{i_{1} \ldots i_{r}}^{(r)}\right\|_{\infty}\left\|F_{i_{r+1} \ldots i_{r+\alpha(j)}}^{(\alpha(j))}\right\|_{\infty}\left\|F_{i_{r+\alpha(j)+1} \ldots i_{n}}^{(n-r-\alpha(j))}\right\|_{\infty} \\
& \leq M\|F^{(\alpha \cdots j} \underbrace{(\alpha(j))}_{\alpha(j)}\|_{\infty} M=M^{2}\left\|\prod_{k=1}^{\alpha(j)} F_{j}\left(\psi_{j}^{k-1}\right)\right\|_{\infty} \\
& <M^{2} \frac{1}{2 M^{2}}=\frac{1}{2},
\end{aligned}
$$

which is a contradiction. 
REMARK 4.3. The proof of Lemma 4.15 directly shows that in particular when

$$
i_{s} \ldots i_{t}=\underbrace{j \ldots j}_{t-s+1}
$$

for some $j \in\left\{1, \ldots, N_{*}\right\}$, we have $t-s+1<\alpha(j)$.

It is not difficult to show the following result.

LEMMA 4.16. For any $n \geq 1$ and for any given index $i_{1} \ldots i_{n}$, there exists a (unique) partition of $\{1, \ldots, n\}$ such that

(1) $1=n_{0} \leq n_{1}<\ldots<n_{k} \leq n_{k+1}=n$ for some $k \geq 0$

(2) either $n_{1}=1$ or $i_{1} \ldots i_{n_{1}}$ is increasing with $i_{n_{1}-1}<i_{n_{1}}$;

(3) for each $l=1, \ldots, k-1, i_{n_{l}} \ldots i_{n_{l+1}}$ is bowl-shaped;

(4) $i_{n_{k}} \ldots i_{n}$ is decreasing.

Definition 4.2. For a given index $\omega=i_{1} \ldots i_{n}, n \geq 3, b(\omega)$ denotes the number of bowl-shaped subindices contained in $\omega$, i.e., $b(\omega)=\max \{k-1,0\}$ with the notation as above.

For each $n \geq 1$, define $\mathcal{U}_{n}$ to be

$$
\mathcal{U}_{n}=\left\{i_{1} \ldots i_{n} \in \mathcal{I}_{\beta} \mid\left\|F_{i_{1} \ldots i_{n}}^{(n)}\right\|_{\infty} \geq 1\right\}
$$

and let $\mathcal{U}=\bigcup_{n=1}^{\infty} \mathcal{U}_{n}$.

Lemma 4.17. Suppose the assumption in Theorem 4.10 holds and that there exists $\left\{n_{k}\right\}_{k=1}^{\infty}$ for which $n_{k} \rightarrow \infty$ as $k \rightarrow \infty$ and $\mathcal{U}_{n_{k}} \neq \emptyset$. Then for any given $L \geq 1$, there exists $\omega \in \mathcal{U}$ such that $b(\omega) \geq L$.

Proof. We first show that there exists $D \geq 1$ for which for any $n \geq 1$ and for any given $\omega=i_{1} \ldots i_{n} \in \mathcal{U}_{n}$, there exists a (unique) partition of $\{1, \ldots, n\}$ such that

(1) $1=n_{0} \leq n_{1}<\ldots<n_{k} \leq n_{k+1}=n$ for some $k \geq 0$;

(2) either $n_{1}=1$ or $i_{1} \ldots i_{n_{1}}$ is increasing with $i_{n_{1}-1}<i_{n_{1}}$;

(3) for each $l=1, \ldots, k-1, i_{n_{l}} \ldots i_{n_{l+1}}$ is bowl-shaped;

(4) $i_{n_{k}} \ldots i_{n}$ is decreasing;

(5) for each $l=0,1, \ldots, k, n_{l+1}-n_{l} \leq D$.

By Lemma 4.16, it suffices to show (5). In fact, it immediately follows from Lemma 4.15 that $n_{1}-n_{0}=n_{1}-1<\alpha$ and $n_{k+1}-n_{k}=n-n_{k}<\alpha$, since each corresponding index is monotone. Also, for each $l=1, \ldots, k-1$, we have $n_{l+1}-n_{l}<\alpha+N_{*}$, since each index $i_{n_{l}} \ldots i_{n_{l+1}}$ consists of one decreasing index and one strictly increasing index. Letting $D=\alpha+N_{*}-1$, we obtain $n_{l+1}-n_{l} \leq D$ for each $l=0,1, \ldots, k$. 
Now choose $n \geq(L+3) D$ and $\omega=i_{1} \ldots i_{n} \in \mathcal{U}_{n}$. Using the notations as above, we get

$$
(L+3) D \leq n=\sum_{l=0}^{k}\left(n_{l+1}-n_{l}\right)+1 \leq(k+1) D+1,
$$

which implies that $b(\omega)=k-1 \geq L$.

REMARK 4.4. In case $k \geq 1$, it is easy to see that

$$
b(\omega)>n / D-2 .
$$

The following is true for any interval map that satisfies condition (C1).

LEMMA 4.18. Let $j_{1} \ldots j_{m}, m \geq 3$, be bowl-shaped, i.e., there exists $r$, $2 \leq r \leq m-1$, such that $j_{1}>j_{r}$ and

$$
j_{1} \geq \ldots \geq j_{r}<j_{r+1}<\ldots<j_{m} .
$$

Let $z_{m+1} \in(0,1]$ be given and for each $s=1, \ldots, m$, let

(1) $z_{s}=\psi_{j_{s}} \circ \ldots \circ \psi_{j_{m}}\left(z_{m+1}\right)=\psi_{j_{s}}\left(z_{s+1}\right)\left(z_{s} \in I_{j_{s}}\right)$;

(2) $A_{s}=F_{j_{s}}\left(z_{s+1}\right)$.

Then for each $s=1, \ldots, m$,

$$
A_{s} \leq z_{s} / z_{s+1}
$$

Moreover, if for some $t \in\{1, \ldots, r-1\}$,

$$
j_{1} \geq \ldots \geq j_{t}>j_{t+1}=\ldots=j_{r}<j_{r+1}
$$

(such a $t$ always exists), then

$$
A_{t} \leq z_{t} / z_{t+1}-A_{r}
$$

Proof. First, note that for each $s=1, \ldots, m$, we have $z_{s+1}>0$. Then for a fixed $s, 1 \leq s \leq m$,

$$
\begin{aligned}
z_{s} & =\psi_{j_{s}}\left(z_{s+1}\right)=\int_{0}^{z_{s+1}} F_{j_{s}}(x) d x+a_{j_{s}-1}=\int_{0}^{z_{s+1}} \mathcal{F}_{j_{s}}(x) d x+\int_{z_{s+1}}^{1} \mathcal{F}_{j_{s}-1}(x) d x \\
& \geq \int_{0}^{z_{s+1}} \mathcal{F}_{j_{s}}(x) d x \geq z_{s+1} \mathcal{F}_{j_{s}}\left(z_{s+1}\right) \geq z_{s+1} F_{j_{s}}\left(z_{s+1}\right)=z_{s+1} A_{s},
\end{aligned}
$$

which implies $A_{s} \leq z_{s} / z_{s+1}$.

To prove the second statement, observe that $z_{t+1} \in I_{j_{t+1}}$ and $z_{r+1} \in$ $I_{j_{r+1}}$, so that $z_{t+1} \leq z_{r+1}$, since $j_{t+1}<j_{r+1}$. Noticing that $j_{t}>j_{r}$, we have $\mathcal{F}_{j_{t}} \geq \mathcal{F}_{j_{r}}+F_{j_{t}}$, which means

$$
\mathcal{F}_{j_{t}}\left(z_{t+1}\right) \geq \mathcal{F}_{j_{r}}\left(z_{t+1}\right)+F_{j_{t}}\left(z_{t+1}\right) \geq \mathcal{F}_{j_{r}}\left(z_{r+1}\right)+F_{j_{t}}\left(z_{t+1}\right) \geq A_{r}+A_{t} .
$$


Thus similarly

$$
z_{t}=\psi_{j_{t}}\left(z_{t+1}\right) \geq \int_{0}^{z_{t+1}} \mathcal{F}_{j_{t}}(x) d x \geq z_{t+1} \mathcal{F}_{j_{t}}\left(z_{t+1}\right) \geq z_{t+1}\left(A_{r}+A_{t}\right)
$$

Therefore $A_{t} \leq z_{t} / z_{t+1}-A_{r}$.

Proof of Theorem 4.10. Let $\omega=i_{1} \ldots i_{n} \in \mathcal{U}_{n}, n \geq 3 \alpha$, and choose $x^{*} \in[0,1]$ so that $F_{i_{1} \ldots i_{n}}^{(n)}\left(x^{*}\right) \geq 1 / 2$. Denote the partition of $\{1, \ldots, n-\alpha(1)\}$ given in Lemma 4.16 by

$$
1=n_{0} \leq n_{1}<\ldots<n_{k} \leq n_{k+1}=n-\alpha(1), \quad k \geq 2 .
$$

Note that $b(\omega) \leq k-1+\alpha(1)$. Let $x_{n+1}=x^{*}$ and for each $s=1, \ldots, n$, let

$$
x_{s}=\psi_{i_{s}} \circ \ldots \circ \psi_{i_{n}}\left(x^{*}\right)=\psi_{i_{s}}\left(x_{s+1}\right) \text {. }
$$

Since $i_{n-\alpha(1)+1} \ldots i_{n} \neq 1 \ldots 1$ (see Remark 4.3), we have $x_{s}>0$ for each $s=1, \ldots, n-\alpha(1)+1$. Also for each $l=2, \ldots, k, x_{n_{l}} \geq a_{1}$, since $i_{n_{l}}>$ $i_{n_{l}-1} \geq 1$.

Fix $l, 1 \leq l \leq k-1$, and consider a bowl-shaped index $j_{1} \ldots j_{m}=$ $i_{n_{l}} \ldots i_{n_{l+1}}$, where $m=n_{l+1}-n_{l}+1$. Let $z_{m+1}=x_{n_{l+1}+1}(>0)$ and for each $s=1, \ldots, m$, define $z_{s}=\psi_{j_{s}}\left(z_{s+1}\right)\left(=\psi_{i_{n_{l}+s-1}}\left(x_{n_{l}+s}\right)\right)$ and $A_{s}=F_{j_{s}}\left(z_{s+1}\right)$. Under the notations in Lemma 4.18, observe that for each $s=1, \ldots, m$,

$$
A_{s} \leq \frac{z_{s}}{z_{s+1}}
$$

and

$$
A_{t} \leq \frac{z_{t}}{z_{t+1}}-A_{r}=\left(1-\frac{z_{t+1}}{z_{t}} A_{r}\right) \frac{z_{t}}{z_{t+1}}
$$

We now show that there exists $\delta>0$, which depends on $T$ only, such that

$$
\frac{z_{t+1}}{z_{t}} A_{r} \geq \delta>0
$$

First it follows from Lemma 4.13 that for each $s=1, \ldots, m$,

$$
A_{s} \geq \frac{1}{2 M^{2}}
$$

Consequently,

$$
z_{t+1} \geq A_{t+1} z_{t+2} \geq \ldots \geq A_{t+1} A_{t+2} \ldots A_{r} z_{r+1} \geq\left(\frac{1}{2 M^{2}}\right)^{r-t} z_{r+1} .
$$

Using the notation in the proof of Lemma 4.17, we have $r-t \leq m-2 \leq D-1$. Also using the fact that $z_{r+1} \in I_{j_{r+1}}$ and $j_{r+1}>j_{r} \geq 1$, we get $z_{r+1} \geq a_{1}$ and so

$$
z_{t+1} \geq\left(\frac{1}{2 M^{2}}\right)^{D-1} a_{1}
$$


Let $\delta=\left(2 M^{2}\right)^{-D} a_{1}>0$. Then

$$
\frac{z_{t+1}}{z_{t}} A_{r} \geq\left(\frac{1}{2 M^{2}}\right)^{D-1} a_{1} \frac{1}{2 M^{2}}=\delta .
$$

This indicates that

$$
A_{t} \leq(1-\delta) \frac{z_{t}}{z_{t+1}}
$$

which combined with (4.4) implies that for a fixed $l, 1 \leq l \leq k-1$,

$$
\begin{aligned}
F_{i_{n_{l} \ldots i_{l+1}-1}}^{\left(n_{l+1}-n_{l}\right)}\left(x_{n_{l+1}}\right) & =F_{j_{1} \ldots j_{m-1}}^{(m-1)}\left(z_{m}\right)=\prod_{s=1}^{m-1} A_{s} \leq(1-\delta) \prod_{s=1}^{m-1} \frac{z_{s}}{z_{s+1}} \\
& =(1-\delta) \frac{z_{1}}{z_{m}}=(1-\delta) \frac{x_{n_{l}}}{x_{n_{l+1}}} .
\end{aligned}
$$

Therefore

$$
\begin{aligned}
\frac{1}{2} & \leq F_{i_{1} \ldots i_{n}}^{(n)}\left(x^{*}\right)=F_{i_{1} \ldots i_{n_{1}-1}}^{\left(n_{1}-1\right)}\left(x_{n_{1}}\right)\left(\prod_{l=1}^{k-1} F_{i_{n_{l} \ldots i_{n_{l+1}}}}^{\left(n_{l+1}-n_{l}\right)}\left(x_{n_{l+1}}\right)\right) F_{i_{n_{k}} \ldots i_{n}}^{\left(n-n_{k}+1\right)}\left(x^{*}\right) \\
& \leq M \prod_{l=1}^{k-1}\left((1-\delta) \frac{x_{n_{l}}}{x_{n_{l+1}}}\right) M \leq M^{2} \frac{x_{n_{1}}}{x_{n_{k}}}(1-\delta)^{k-1} \\
& \leq M^{2} \frac{1}{a_{1}}(1-\delta)^{b(\omega)-\alpha(1)}
\end{aligned}
$$

where we have used the fact that for each $l=2, \ldots, k, x_{n_{l}} \geq a_{1}$, since $i_{n_{l}}>i_{n_{l}-1} \geq 1$.

This gives a contradiction if $n$ is chosen so that

$$
\frac{n}{D}>\frac{\ln \left(a_{1} /\left(2 M^{2}\right)\right)}{\ln (1-\delta)}+2+\alpha(1),
$$

which would imply that

$$
b(\omega)-\alpha(1)>\frac{n}{D}-2-\alpha(1)>\frac{\ln \left(a_{1} / 2 M^{2}\right)}{\ln (1-\delta)}
$$

(see Remark 4.4) or equivalently

$$
M^{2} \frac{1}{a_{1}}(1-\delta)^{b(\omega)-\alpha(1)}<\frac{1}{2} .
$$

Therefore there exists $L \geq 1$ such that for any $n \geq L, \mathcal{U}_{n}=\emptyset$.

5. Ergodic properties of an invariant measure: case II. Throughout this section $T$ is assumed to satisfy the conditions of Theorem 1.1. As mentioned in Remark 4.2, when $F_{N_{*}}(\beta)=1$, every power of the map $T$ fails to be expanding on $[0, \beta]$. However it turns out that $\left(T, g_{\beta} d m\right)$ is exact. 
Theorem 5.1. Suppose $T$ satisfies the conditions in Theorem 1.1 and $F_{N_{*}}(\beta)=1$. If $f \in \mathrm{BV}(\beta), f \geq 0$, and $\|f\|=\beta$, then

$$
\left\|P^{n} f-\chi_{[0, \beta]}\right\| \rightarrow 0 \quad \text { as } n \rightarrow \infty .
$$

Notice that the assumption $F_{N_{*}}(\beta)=1$ implies $P \chi_{[0, \beta]}=\chi_{[0, \beta]}$. Let $g_{*}=\chi_{[0, \beta]} / \beta \in \mathrm{BV}(\beta)$. Theorem 5.1 shows that $\left(T, g_{*} d m\right)$ is exact (see the proof of Theorem 4.5), so that $g_{\beta}=g_{*}=\chi_{[0, \beta]} / \beta$. Combined with Theorem 4.5 , this yields the following.

Corollary 5.2. Suppose $T$ satisfies the conditions in Theorem 1.1. Then $g_{\beta}$ obtained from Lemma 4.4 is a unique invariant density of $T$ in $\mathrm{BV}(\beta)$ and $\left(T, g_{\beta} d m\right)$ is exact.

In order to show Theorem 5.1, we establish convergence at the single point zero, after which the full result will follow easily.

Lemma 5.3. Let $f \in \mathcal{J}(\beta)$. Then $\left(P^{n} f\right)(0) \rightarrow\|f\|$ as $n \rightarrow \infty$.

Proof. Since we assume $P \chi_{[0, \beta]}=\chi_{[0, \beta]}$, using Remark 2.1, we may further assume that $\mathcal{F}_{N_{*}} \equiv 1$.

For each $n \geq 0$, let $C_{n}=\left(P^{n} f\right)(0)$. Then

$$
C_{n+1}=\sum_{i=1}^{N} F_{i}(0)\left(P^{n} f\right)\left(\psi_{i}(0)\right) \leq \sum_{i=1}^{N} F_{i}(0)\left(P^{n} f\right)(0)=C_{n},
$$

so that as $n \rightarrow \infty, C_{n} \searrow C_{*}$ for some $C_{*} \geq\|f\|$.

Let $b_{0}=0$. For each $k \geq 1$, let $l_{k}=\max \left\{i \mid 1 \leq i \leq N_{*}, F_{i}\left(b_{k-1}\right)>0\right\}$ and $b_{k}=\psi_{l_{k}}\left(b_{k-1}\right)$.

Next, we claim that for each $k, \lim _{n \rightarrow \infty} P^{n} f\left(b_{k}\right)=\lim _{n \rightarrow \infty} P^{n} f\left(b_{0}\right)$ $=C_{*}$.

Once again we use $(\mathrm{C} 1)$ for a fixed $k$ to find

$$
F_{l_{k}}\left(b_{k-1}\right)=1-\mathcal{F}_{l_{k}-1}\left(b_{k-1}\right)>0,
$$

while for each $n \geq 0$,

$$
\begin{aligned}
\left(P^{n+1} f\right)\left(b_{k-1}\right) & =\sum_{i=1}^{N_{*}} F_{i}\left(b_{k-1}\right)\left(P^{n} f\right)\left(\psi_{i}\left(b_{k-1}\right)\right) \\
& =\sum_{i=1}^{l_{k}} F_{i}\left(b_{k-1}\right)\left(P^{n} f\right)\left(\psi_{i}\left(b_{k-1}\right)\right) \\
& \leq \sum_{i=1}^{l_{k}-1} F_{i}\left(b_{k-1}\right)\left(P^{n} f\right)(0)+F_{l_{k}}\left(b_{k-1}\right)\left(P^{n} f\right)\left(\psi_{l_{k}}\left(b_{k-1}\right)\right) \\
& =\sum_{i=1}^{l_{k}-1} F_{i}\left(b_{k-1}\right) C_{n}+F_{l_{k}}\left(b_{k-1}\right)\left(P^{n} f\right)\left(b_{k}\right) .
\end{aligned}
$$


Thus for each $k \geq 1$,

$$
\liminf _{n \rightarrow \infty}\left(P^{n} f\right)\left(b_{k-1}\right) \leq\left(1-F_{l_{k}}\left(b_{k-1}\right)\right) C_{*}+F_{l_{k}}\left(b_{k-1}\right) \liminf _{n \rightarrow \infty}\left(P^{n} f\right)\left(b_{k}\right) .
$$

Noticing that $\lim _{n \rightarrow \infty}\left(P^{n} f\right)\left(b_{0}\right)=C_{*}$ and using induction on $k$, we have for each $k \geq 0$,

$$
C_{*} \leq \liminf _{n \rightarrow \infty}\left(P^{n} f\right)\left(b_{k}\right) .
$$

Meanwhile, fix $k \geq 0$. Since for any given $n \geq 0, P^{n} f$ is decreasing, it follows that $\left(P^{n} f\right)\left(b_{k}\right) \leq\left(P^{n} f\right)(0)=C_{n}$, which implies

$$
\limsup _{n \rightarrow \infty}\left(P^{n} f\right)\left(b_{k}\right) \leq C_{*} \text {. }
$$

Thus for each $k \geq 0, \lim _{n \rightarrow \infty}\left(P^{n} f\right)\left(b_{k}\right)=C_{*}$, as claimed.

Next, observe that for each $k \geq 0, b_{k}<\beta$, for if not, and if $k$ is chosen to be minimal such that $b_{k}=\beta$, then obviously $l_{k}=N_{*}$ and $F_{N_{*}}\left(b_{k-1}\right)>0$. However, $F_{N_{*}}=1-\mathcal{F}_{N_{*}-1}$ is increasing, so $F_{N_{*}}>0$ on $\left[b_{k-1}, b_{k}\right]=\left[b_{k-1}, \beta\right]$, contradicting $\psi_{N_{*}} \equiv \beta$ on $\left[b_{k-1}, \beta\right]$ in the convexity condition $(\mathrm{C} 1)$.

However, we will show that

$$
\sup \left\{b_{k} \mid k \geq 0\right\}=\beta,
$$

in which case for any $x \in[0, \beta), \lim _{n \rightarrow \infty}\left(P^{n} f\right)(x)=C_{*}$. As $\lim _{n \rightarrow \infty}\left(P^{n} f\right)(x)$ $=0$ on $(\beta, 1]$, it easily follows that $C_{*}=\|f\|$, i.e., $\lim _{n \rightarrow \infty}\left(P^{n} f\right)(x)=\|f\|$ on $[0, \beta]$. In particular, $\left(P^{n} f\right)(0) \rightarrow\|f\|$ as $n \rightarrow \infty$, which completes the proof.

To see (5.1), let $r=\max \left\{l_{k} \mid k \geq 1\right\}$ and notice that $l_{1} \geq 2$ so $r \geq 2$. If $r<N_{*}$ set $s=\inf \left\{T\left(x^{+}\right) \mid a_{r} \leq x<\beta\right\}$, otherwise set $s=\beta$. We want to show that the sequence $b_{k}$ is contained in $[0, s]$. If for some $k, b_{k}>s$, then $r<N_{*}$ and $\sum_{i=r+1}^{N_{*}} F_{i}\left(b_{k}\right)>0$. If this is not the case, i.e. $\sum_{i=r+1}^{N_{*}} F_{i}\left(b_{k}\right)=0$, then $\sum_{i=r+1}^{N_{*}} F_{i} \equiv 0$ on $\left[s, b_{k}\right]$ since the sum is an increasing function on $[0, \beta]$, which in view of the convexity condition $(\mathrm{C} 1)$ contradicts the definition of $s$ above. But then clearly $l_{k+1}>r$, another contradiction.

Choose $m \geq 1$ so that $l_{m}=r$. Then $b_{m}=\psi_{l_{m}}\left(b_{m-1}\right)=\psi_{r}\left(b_{m-1}\right) \in$ $\left[a_{r-1}, a_{r}\right]$. Since $0<b_{m}<\beta$ and

$$
T\left[b_{m}, \beta\right] \subseteq\left[T_{r}\left(b_{m}\right), \beta\right] \cup[s, \beta] \subseteq\left[T_{r}\left(b_{m}\right), \beta\right] \cup\left[b_{m}, \beta\right],
$$

it follows from Lemma 4.2 that $b_{m-1}=T_{r}\left(b_{m}\right)<b_{m}$, i.e., $T_{r}\left(a_{r-1}\right) \leq$ $T_{r}\left(b_{m}\right)<b_{m} \leq s$.

Now, on $[0, s], F_{r}$ is increasing, so

$$
0<F_{r}\left(b_{m-1}\right)=F_{r}\left(T_{r}\left(b_{m}\right)\right) \leq F_{r}\left(b_{m}\right),
$$

which implies $l_{m+1}=r$. A simple induction shows that $l_{m+k}=r$ for all $k=0,1, \ldots$ and that the sequence $b_{m+k}$ is increasing on $\left[a_{r-1}, a_{r}\right]$ for all such $k$. 
Define $b^{*}=\lim _{k \rightarrow \infty} b_{m+k}=\lim _{l \rightarrow \infty} b_{l}$. Using $a_{r-1} \leq b^{*} \leq a_{r}$ and $b^{*} \leq s$, combined with $T_{r}\left(b^{*}\right)=b^{*}$ yields

$$
T\left[b^{*}, \beta\right] \subseteq\left[T_{r}\left(b^{*}\right), \beta\right] \cup[s, \beta] \subseteq\left[b^{*}, \beta\right] .
$$

Finally, Lemma 4.2 implies $b^{*}=\beta$ and (5.1) has been verified.

Proof of Theorem 5.1. Let $f \in \mathcal{J}(\beta)$ and $\|f\|=\beta$. For each $n \geq 1$, let $d_{n}=\inf \left\{x \in[0,1] \mid\left(P^{n} f\right)(x) \leq 1\right\}$. Clearly, for any given $n \geq 1, d_{n}(\leq \beta)$ exists, and

$$
\beta=\left\|P^{n} f\right\|=\int_{0}^{d_{n}} P^{n} f+\int_{d_{n}}^{1} P^{n} f
$$

which implies that

$$
\begin{aligned}
\int_{d_{n}}^{1}\left|P^{n} f-\chi_{[0, \beta]}\right| & =\int_{d_{n}}^{\beta}\left[1-\left(P^{n} f\right)(x)\right] d x \\
& =\int_{0}^{d_{n}}\left[\left(P^{n} f\right)(x)-1\right] d x=\int_{0}^{d_{n}}\left|P^{n} f-\chi_{[0, \beta]}\right| .
\end{aligned}
$$

Since for each $n \geq 1, P^{n} f$ is decreasing, we have

$$
\left\|P^{n} f-\chi_{[0, \beta]}\right\|=2 \int_{0}^{d_{n}}\left|P^{n} f-\chi_{[0, \beta]}\right| \leq 2 d_{n}\left[\left(P^{n} f\right)(0)-1\right] \leq 2\left[\left(P^{n} f\right)(0)-1\right] .
$$

It follows from Lemma 5.3 that $\left\|P^{n} f-\chi_{[0, \beta]}\right\| \rightarrow 0$ as $n \rightarrow \infty$.

Now suppose $f \in \operatorname{BV}(\beta), f \geq 0$, and $\|f\|=\beta$. Then $f-\chi_{[0, \beta]} \in \mathrm{BV}_{0}(\beta)$, and hence there exist $f^{1}, f^{2} \in \mathcal{J}(\beta)$ such that $f-\chi_{[0, \beta]}=f^{1}-f^{2}$ and $\left\|f^{1}\right\|=\left\|f^{2}\right\|$. Thus

$$
\begin{aligned}
& \left\|P^{n} f-\chi_{[0, \beta]}\right\|=\left\|P^{n}\left(f^{1}-f^{2}\right)\right\| \\
& \quad=\left\|\left(P^{n} f^{1}-\left\|f^{1}\right\| \chi_{[0, \beta]} / \beta\right)-\left(P^{n} f^{2}-\left\|f^{2}\right\| \chi_{[0, \beta]} / \beta\right)\right\| \\
& \quad \leq\left[\left\|P^{n}\left(\beta f^{1} /\left\|f^{1}\right\|\right)-\chi_{[0, \beta]}\right\|+\left\|P^{n}\left(\beta f^{2} /\left\|f^{2}\right\|\right)-\chi_{[0, \beta]}\right\|\right] \cdot\left\|f^{1}\right\| / \beta,
\end{aligned}
$$

where the last expression converges to 0 as $n \rightarrow \infty$. Therefore $\left\|P^{n} f-\chi_{[0, \beta]}\right\|$ $\rightarrow 0$ as $n \rightarrow \infty$.

6. Ergodic properties on the unit interval. Suppose $T$ satisfies the conditions of Theorem 1.1 and $\bigcup_{n=1}^{\infty} T^{n}\left[0, a_{1}\right]=T[0,1]$ (which is the case in particular when $\beta=1$ ). Corollary 5.2 indicates that $g \in \mathrm{BV}$ obtained from Theorem 1.1 is a unique invariant density of $T$ and $(T, g d m)$ is exact. Furthermore, Theorem 4.5, combined with Theorem 4.10, shows that if $F_{N}(1)<1$, then $(T, g d m)$ has exponential decay of correlations and some power of $T$ is expanding, hence Bernoulli. In this section, we will consider the 
case where $\bigcup_{n=1}^{\infty} T^{n}\left[0, a_{1}\right] \subsetneq T[0,1]$ (so $\beta<1$ ) and investigate ergodic properties of $(T, g d m)$ on the unit interval. Maps satisfying the Lasota-Yorke convexity condition are known to have the property that the invariant probability density is unique and the unique a.c.i.m. is exact for $T$ (cf. [9]). It turns out that our weaker convexity condition (C) is not sufficient to imply exactness, or even to guarantee uniqueness of the invariant probability density as the following simple example shows.

EXAMPLE 6.1. Let $T(x)= \begin{cases}2 x & \text { if } 0 \leq x<1 / 4 \\ 2 x-1 / 2 & \text { if } 1 / 4 \leq x<3 / 4, \\ 2 x-1 & \text { if } x \geq 3 / 4\end{cases}$

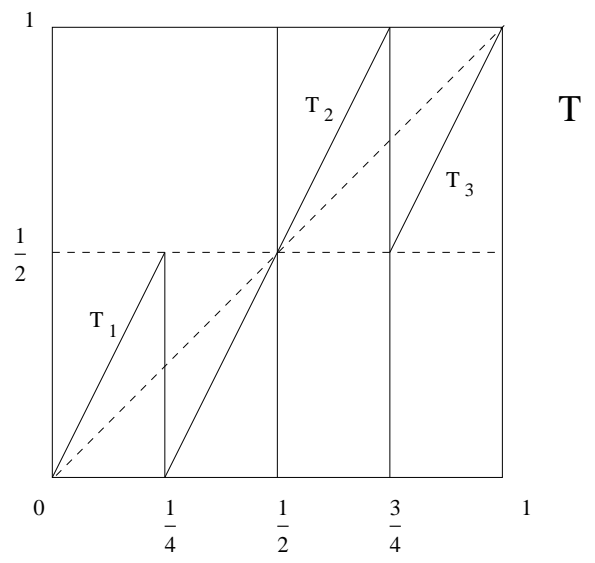

Fig. 6.1

(see Figure 6.1). Lebesgue measure is preserved and $\beta=1 / 2$. However, $T$ supports infinitely many a.c.i.m. on $[0,1]$ with densities $g_{1}=2 \chi_{[0,1 / 2]}, g_{2}=$ $2 \chi_{[1 / 2,1]}$ and $g_{\alpha}=\alpha g_{1}+(1-\alpha) g_{2}$, for $0<\alpha<1$, so $T$ is certainly not exact. (However, $T_{\beta}$ is exact, as required by the arguments in $\S 5$, and obviously $\left.T\right|_{[1 / 2,1]}$ is also exact.) Consider the nontrivial invariant interval $[1 / 2,1]$. If it is only noticed that $T[1 / 2,1] \subseteq[1 / 2,1]$ then $T^{-1}[1 / 2,1] \supseteq[1 / 2,1]$, from which it follows that the interval is invariant. This simple observation turns out to be the key to understanding exactness, even when Lebesgue measure is not invariant.

With this example in mind, we define our mixing condition denoted by $(\mathrm{M})$ :

(M) For each $d \in(\beta, 1], T[\beta, d] \nsubseteq[\beta, d]$.

REMARK 6.1. We present two conditions related to the uniqueness of the invariant density.

(A) For any $c, d, 0<c<d$, we have $0 \in \bigcap_{n \geq 1} \bigcup_{k \geq n} T^{k}[c, d]$. 
(B) If $D$ is a finite union of closed intervals, then either $0 \in D$ or $T(D)$ $\nsubseteq D$.

It can be shown directly that $(\mathrm{A})$ is a necessary and sufficient condition for $T$ to have a unique invariant density in BV. It is clear that (A) implies (B), which is stronger than (M). In general, neither of the converses is true. However, it turns out that condition (M), together with the convexity condition (C), does imply (A). In other words, it guarantees the uniqueness of invariant density, and in fact the exactness also follows.

Throughout this section $T$ is assumed to satisfy the conditions of Theorem 1.1 and condition (M).

Lemma 6.1. Let $g_{\beta}$ be the invariant density defined in Lemma 4.4. Then $g_{\beta}=g$.

Proof. Let $g_{2}=g \chi_{(\beta, 1]}=g-g_{\beta}$. Since $P g_{\beta}=g_{\beta}$, it follows that $P g_{2}=g_{2}$. Suppose $g_{2} \not \equiv 0$. Since $g_{2}$ is decreasing on $(\beta, 1]$, there exists $\gamma \in(\beta, 1]$ such that $\overline{g_{2}^{-1}(\mathbb{R} \backslash\{0\})}=[\beta, \gamma]$. We claim that $T[\beta, \gamma] \subseteq[\beta, \gamma]$, which contradicts the mixing condition $(\mathrm{M})$ and so yields $g_{2} \equiv 0$, i.e., $g_{\beta}=g$. To prove the claim, let $B=[0, \beta) \cup(\gamma, 1]$ and observe

$$
0=\int_{B} g_{2} d m=\int P g_{2} \cdot \chi_{B} d m=\int_{T^{-1} B} g_{2} d m=\int_{B^{\mathrm{c}} \cap T^{-1} B} g_{2} d m,
$$

which implies that $B^{\mathrm{c}} \cap T^{-1} B \subseteq\{\beta, \gamma\}$. Since $T\left(B^{\mathrm{c}}\right)=T[\beta, \gamma]$ is a finite union of nontrivial closed intervals, it follows that $T\left(B^{\mathrm{c}}\right) \subseteq B^{\mathrm{c}}$, as we have claimed.

LEMma 6.2. For a given $f \in \mathrm{BV}$,

$$
\left\|\left(P^{n} f\right) \chi_{(\beta, 1]}\right\| \rightarrow 0 \quad \text { as } n \rightarrow \infty \text {. }
$$

Proof. For each $n \geq 1$, let $h_{n}=(1 / n) \sum_{s=0}^{n-1} P^{s} \mathbf{1}$ and take a subsequence $\left\{n_{k}\right\}_{k=1}^{\infty}$ so that $h_{n_{k}} \rightarrow h$ in $\|\cdot\|$ as $n_{k} \rightarrow \infty$, where $h \in \mathcal{J}$ is an invariant density of $T$ (see the proof of Theorem 1.1). It follows from Lemma 6.1 that $h(x)=0$ on $(\beta, 1]$.

Let $f \geq 0$ and $f \in \mathrm{BV}$. Since $T[0, \beta] \subseteq[0, \beta]$, we have $T^{-1}[\beta, 1] \subseteq[\beta, 1]$ and so

$$
\int_{\beta}^{1} \operatorname{Pf} d m \leq \int_{\beta}^{1} f d m
$$

Let $M=\|f\|_{\infty}<\infty$. It inductively follows that for a given $n \geq 1$,

$$
\int_{\beta}^{1} P^{n-1} f d m \leq \int_{\beta}^{1} P^{n-2} f d m \leq \ldots \leq \int_{\beta}^{1} f d m
$$


which implies

$$
\begin{aligned}
\int_{\beta}^{1} P^{n-1} f d m & =\frac{1}{n} \sum_{s=0}^{n-1} \int_{\beta}^{1} P^{n-1} f d m \leq \frac{1}{n} \sum_{s=0}^{n-1} \int_{\beta}^{1} P^{s} f d m \\
& \leq \frac{1}{n} \sum_{s=0}^{n-1} \int_{\beta}^{1} P^{s}(M \cdot \mathbf{1}) d m=M \frac{1}{n} \sum_{s=0}^{n-1} \int_{\beta}^{1} P^{s} \mathbf{1} d m \\
& =M\left\|h_{n} \chi_{(\beta, 1]}\right\| \leq M\left\|h_{n}-h\right\| .
\end{aligned}
$$

Since $\left\|h_{n_{k}}-h\right\| \rightarrow 0$ as $n_{k} \rightarrow \infty$, it follows that

$$
\left\|\left(P^{n} f\right) \chi_{(\beta, 1]}\right\| \rightarrow 0 \quad \text { as } n \rightarrow \infty \text {. }
$$

For any given $f \in \mathrm{BV}$, letting $f=f_{+}-f_{-}$, where $f_{+}, f_{-} \geq 0$ and $f_{+}, f_{-} \in \mathrm{BV}$, and applying the same argument to $f_{+}, f_{-}$, we have

$$
\left\|\left(P^{n} f\right) \chi_{(\beta, 1]}\right\| \rightarrow 0 \quad \text { as } n \rightarrow \infty \text {. }
$$

Corollary 6.3. Let $g_{\beta}$ be the invariant density defined in Lemma 4.4. Then $g_{\beta}=g$ is a unique invariant density of $T$.

Proof. Suppose $\phi \in \mathrm{BV}, \phi \geq 0$, and $P \phi=\phi$. By Lemma 6.2,

$$
\int_{\beta}^{1} \phi d m=\int_{\beta}^{1} P^{m} \phi d m \rightarrow 0 \quad \text { as } m \rightarrow \infty,
$$

which implies $\phi(x)=0$ on $(\beta, 1]$, i.e., $\phi \in \mathrm{BV}(\beta)$. From Corollary 5.2, we get $\phi=g$. Therefore $g=g_{\beta}$ is a unique invariant density of $T$.

THEOREM 6.4. Suppose $T$ satisfies the conditions in Theorem 1.1 and condition (M). Then $(T, g d m)$ is exact.

Proof. Let $f \in \mathrm{BV}, f \geq 0$, and $\|f\|=1$. Let $\varepsilon>0$ be given. From Lemma 6.2, there exists $m \geq 1$ such that

$$
\left\|\left(P^{m} f\right) \chi_{(\beta, 1]}\right\|<\varepsilon / 3 \text {. }
$$

Let $f_{\beta}=\left(P^{m} f\right) \chi_{[0, \beta]} \in \mathrm{BV}(\beta)$ and $A=\left\|f_{\beta}\right\|$. Using Theorem 4.5, choose $n>m$ so that

$$
\left\|P^{n-m}\left(f_{\beta} / A\right)-g\right\|<\varepsilon / 3 .
$$

Since $A=1-\left\|\left(P^{m} f\right) \chi_{(\beta, 1]}\right\| \leq 1$ and $P$ is a contraction in $\|\cdot\|$,

$$
\begin{aligned}
\left\|P^{n} f-g\right\| & \leq\left\|P^{n-m} f_{\beta}-A g\right\|+\|(A-1) g\|+\left\|P^{n-m}\left(P^{m} f-f_{\beta}\right)\right\| \\
& \leq\left\|P^{n-m}\left(f_{\beta} / A\right)-g\right\|+(1-A)+\left\|\left(P^{m} f\right) \chi_{(\beta, 1]}\right\| \\
& <\varepsilon / 3+\varepsilon / 3+\varepsilon / 3=\varepsilon .
\end{aligned}
$$

Therefore $(T, g d m)$ is exact.

TheOREM 6.5. Suppose $T$ satisfies the conditions in Theorem 1.1 and conditions $(\mathrm{M})$ and $(\mathrm{E})$. If $\lim _{x \rightarrow \beta^{+}} F_{N_{*}+1}(x)<1$, then some power of $T$ is expanding, hence Bernoulli. 
Using the additional hypothesis $\lim _{x \rightarrow \beta^{+}} F_{N_{*}+1}(x)<1$ and the mixing condition (M), we modify the proof of Lemma 4.14 and obtain the following.

Lemma 6.6. Suppose the assumption in Theorem 6.5 holds. Then for each $j=1, \ldots, N$, there exists $\alpha(j) \in \mathbb{N}$ such that

$$
\left\|\prod_{k=1}^{\alpha(j)} F_{j}\left(\psi_{j}^{k-1}\right)\right\|_{\infty}<\frac{1}{2 M^{2}} .
$$

Proof. From the proof of Lemma 4.14, it suffices to show that if $N_{*}<j \leq$ $N$ and $T_{j}\left(z^{*}\right)=z^{*}$ for some $z^{*} \in\left[a_{j-1}, a_{j}\right]\left(z^{*} \geq \beta\right)$, then there exist $\varepsilon>0$ and $w^{*}<z^{*}$ such that for any $y \in\left[w^{*}, 1\right], F_{j}(y) \leq 1-\varepsilon<1$. In fact, in the case where $\mathcal{F}_{j}\left(z^{*}\right)<1$, similarly the fact that $\mathcal{F}_{j}$ is upper semicontinuous completes the proof of the claim. If $\mathcal{F}_{j}\left(z^{*}\right)=1$, then it also follows from (4.3) that $\mathcal{F}_{j}(x)=1$ on $\left[0, z^{*}\right)$. Using $T[0, \beta]=[0, \beta]$ and the Markov property of $P$ we get $\int_{0}^{1} \mathcal{F}_{N_{*}}=\int_{0}^{\beta} \mathcal{F}_{N_{*}}=\beta$ so that $\mathcal{F}_{N_{*}}(x)=1$ on $[0, \beta]$. We conclude that $\left(\mathcal{F}_{j}-\mathcal{F}_{N_{*}}\right)(x)=0$ on $[0, \beta]$. Thus $T\left[\beta, z^{*}\right] \subseteq\left[\beta, z^{*}\right]$. By the mixing condition (M), we get $z^{*}=\beta$ and so $j=N_{*}+1$. Note that $F_{N_{*}+1}(x)=0$ on $[0, \beta]$ and $F_{N_{*}+1}$ is decreasing on $(\beta, 1]$. Since $\lim _{x \rightarrow \beta^{+}} F_{N_{*}+1}(x)<1$, it follows that there exist $\varepsilon>0$ and $w^{*}<z^{*}=\beta$ such that for any $y \in\left[w^{*}, 1\right]$, $F_{j}(y)=F_{N_{*}+1}(y) \leq 1-\varepsilon<1$. We also observe that there can be at most one fixed point in each monotonicity interval. For if $a_{j-1} \leq z_{1}<z_{2} \leq a_{j}$ are two fixed points, then as in the proof of Lemma 4.14 we see that $F_{j} \equiv 1$ on $\left[z_{1}, z_{2}\right]$, from which it follows that $T\left[\beta, z_{2}\right] \subseteq\left[\beta, z_{2}\right]$, contradicting (M). The remainder of the argument follows as in Lemma 4.14. We omit the details.

For the completion of the proof of Theorem 6.5, the rest of the arguments in the proof of Theorem 4.10 can be applied with only a slight modification, e.g., replacing $N_{*}$ and $\mathcal{I}_{\beta}$ with $N$ and $\mathcal{I}$, respectively.

\section{References}

[1] R. Bowen, Equilibrium States and the Ergodic Theory of Anosov Diffeomorphisms, Lecture Notes in Math. 470, Springer, Berlin, 1975.

[2] A. Boyarsky and P. Góra, Laws of Chaos. Invariant Measures and Dynamical Systems in One Dimension, Birkhäuser, 1997.

[3] P. Góra and B. Schmitt, Un exemple de transformation dilatante et $C^{1}$ par morceaux de l'intervalle, sans probabilité absolument continue invariante, Ergodic Theory Dynam. Systems 9 (1989), 101-113.

[4] P. Hulse, Uniqueness and ergodic properties of attractive $\mathcal{G}$-measures, ibid. 11 (1991), $65-77$.

[5] M. Jabłoński and J. Malczak, The rate of convergence of iterates of the FrobeniusPerron operator for piecewise convex transformations, Bull. Polish Acad. Sci. Math. 31 (1983), 249-254.

[6] -, - A central limit theorem for piecewise convex transformations of the unit interval, Colloq. Math. 49 (1984), 59-65. 
[7] A. Lasota and M. Mackey, Chaos, Fractals, and Noise. Stochastic Aspects of Dynamics, Springer, New York, 1991.

[8] A. Lasota and J. Yorke, On the existence of invariant measures for piecewise monotonic transformations, Trans. Amer. Math. Soc. 186 (1973), 481-488.

[9] -, -, Exact dynamical systems and the Frobenius-Perron operator, ibid. 273 (1982), $375-384$.

[10] R. Murray, Approximation error for invariant density calculations, Discrete Contin. Dynam. Systems 4 (1998), 535-558.

[11] M. Rychlik, Bounded variation and invariant measures, Studia Math. 76 (1983), 69-80.

Department of Mathematics and Statistics

University of Victoria

P.O. Box 3045

Victoria, B.C., V8W 3P4, Canada

E-mail: cbose@math.uvic.ca

Department of Mathematics

Korea Advanced Institute

of Science and Technology

373-1, Guseong-dong, Yuseong-gu

Daejon, 305-701, South Korea

E-mail:sjs@math.kaist.ac.kr
UMR $5584 \mathrm{du}$ CNRS Laboratoire de Topologie Université de Bourgogne B.P. 400

21011 Dijon Cedex, France E-mail: vmaume@topolog.u-bourgogne.fr schmittb@u-bourgogne.fr

Received October 8, 2001

Revised version February 5, 2002 